\title{
ON PROOFS OF THE FARRELL-JONES CONJECTURE
}

\author{
BARTELS, A.
}

\begin{abstract}
These notes contain an introduction to proofs of Farrell-Jones Conjecture for some groups and are based on talks given in Ohio, Oxford, Berlin, Shanghai, Münster and Oberwolfach in 2011 and 2012.
\end{abstract}

\section{INTRODUCTION}

Let $R$ be a ring and $G$ be a group. The Farrell-Jones Conjecture 25$]$ is concerned with the $K$ - and $L$-theory of the group ring $R[G]$. Roughly it says that the $K$ - and $L$-theory of $R[G]$ is determined by the $K$ - and $L$-theory of the rings $R[V]$ where $V$ varies over the family of virtually cyclic subgroups of $G$ and group homology. The conjecture is related to a number of other conjectures in geometric topology and $K$-theory, most prominently the Borel Conjecture. Detailed discussions of applications and the formulation of this conjecture (and related conjectures) can be found in [10, 32, 33, 34, 35.

These notes are aimed at the reader who is already convinced that the FarrellJones Conjecture is a worthwhile conjecture and is interested in recent proofs 3 , 6, 9 of instances of this Conjecture. In these notes I discuss aspects or special cases of these proofs that I think are important and illustrating. The discussion is based on talks given over the last two years. It will be much more informal than the actual proofs in the cited papers, but I tried to provide more details than I usually do in talks. I took the liberty to express opinion in some remarks; the reader is encouraged to disagree with me. The cited results all build on the seminal work of Farrell and Jones surrounding their conjecture, in particular, their introduction of the geodesic flow as a tool in $K$ - and $L$-theory [23. Nevertheless, I will not assume that the reader is already familiar with the methods developed by Farrell and Jones.

A brief summary of these notes is as follows. Section 1 contains a brief discussion of the statement of the conjecture. The reader is certainly encouraged to consult [10, 32, 33, 34, 35, for much more details, motivation and background. Section2 2 contains a short introduction to geometric modules that is sufficient for these notes. Three axiomatic results, labeled Theorems $\mathrm{A} B$ and $\mathrm{C}$, about the Farrell-Jones Conjecture are formulated in Section 3. Checking for a group $G$ the assumptions of these results is never easy. Nevertheless, the reader is encouraged to find further applications of them. In Section 4 an outline of the proof of Theorem $\mathrm{A}$ is given. Section 5 describes the role of flows in proofs of the Farrell-Jones Conjecture. It also contains a discussion of the flow space for CAT(0)-groups. Finally, in Section6 an application of Theorem $\mathbb{C}$ to some groups of the form $\mathbb{Z}^{n} \rtimes \mathbb{Z}$ is discussed.

Acknowledgement. I had the good fortune to learn from and work with great coauthors on the Farrell-Jones Conjecture; everything discussed here is taken from these cooperations. I thank Daniel Kasprowski, Sebastian Meinert, Adam Mole,

Date: March 2013.

1991 Mathematics Subject Classification. 18F25, 19A31, 19B28, 19G24.

Key words and phrases. Farrell-Jones Conjecture, $K$ - and $L$-theory of group rings. 
Holger Reich, Mark Ullmann and Christoph Winges for helpful comments on an earlier version of these notes. The work described here was supported by the Sonderforschungsbereich 878 - Groups, Geometry \& Actions.

\section{Statement of the Farrell-Jones Conjecture}

Classifying spaces for families. Let $G$ be a group. A family of subgroups of $G$ is a non-empty collection $\mathcal{F}$ of subgroups of $G$ that is closed under conjugation and taking subgroups. Examples are the family Fin of finite subgroups, the family Cyc of cyclic subgroups, the family of virtually cyclic subgroups VCyc, the family $\mathrm{Ab}$ of abelian subgroups, the family $\{1\}$ consisting of only the trivial subgroup and the family All of all subgroups. If $\mathcal{F}$ is a family, then the collection $V \mathcal{F}$ of all $V \subseteq G$ which contain a member of $\mathcal{F}$ as a finite index subgroup is also a family. All these examples are closed under abstract isomorphism, but this is not part of the definition. If $G$ acts on a set $X$ then $\left\{H \leq G \mid X^{H} \neq \emptyset\right\}$ is a family of subgroups.

Definition 1.1. A $G-C W$-complex $E$ is called a classifying space for the family $\mathcal{F}$, if $E^{H}$ is non-empty and contractible for all $H \in \mathcal{F}$ and empty otherwise.

Such a $G$ - $C W$-complex always exists and is unique up to $G$-equivariant homotopy equivalence. We often say such a space $E$ is a model for $E_{\mathcal{F}} G$; less precisely we simply write $E=E_{\mathcal{F}} G$ for such a space.

Example 1.2. Let $\mathcal{F}$ be a family of subgroups. Consider the $G$-set $S:=\coprod_{F \in \mathcal{F}} G / F$. The full simplicial complex $\Delta(S)$ spanned by $S$ (i.e., the simplicial complex that contains a simplex for every non-empty finite subset of $S$ ) carries a simplicial $G$ action. The isotropy groups of vertices of $\Delta(S)$ are all members of $\mathcal{F}$, but for an arbitrary point of $\Delta(S)$ the isotropy group will only contain a member of $\mathcal{F}$ as a finite index subgroup. The first barycentric subdivision of $\Delta(S)$ is a $G$-CW-complex and it is not hard to see that it is a model for $E_{V F} G$.

This construction works for any $G$-set $S$ such that $\mathcal{F}=\left\{H \leq G \mid S^{H} \neq \emptyset\right\}$.

More information about classifying spaces for families can be found in [31].

Statement of the conjecture. The original formulation of the Farrell-Jones Conjecture [25] used homology with coefficients in stratified and twisted $\Omega$-spectra. We will use the elegant formulation of the conjecture developed by Davis and Lück [21. Given a ring $R$ and a group $G$ Davis-Lück construct a homology theory for $G$-spaces

$$
X \mapsto H_{*}^{G}\left(X ; \mathbf{K}_{R}\right)
$$

with the property that $H_{*}^{G}\left(G / H ; \mathbf{K}_{R}\right)=K_{*}(R[H])$.

Definition 1.3. Let $\mathcal{F}$ be a family of subgroups of $G$. The projection $E_{\mathcal{F}} G \rightarrow G / G$ to the one-point $G$-space $G / G$ induces the $\mathcal{F}$-assembly map

$$
\alpha_{\mathcal{F}}: H_{*}^{G}\left(E_{\mathcal{F}} G ; \mathbf{K}_{R}\right) \rightarrow H_{*}^{G}\left(G / G ; \mathbf{K}_{R}\right)=K_{*}(R[G]) .
$$

Conjecture 1.4 (Farrell-Jones Conjecture). For all groups $G$ and all rings $R$ the assembly map $\alpha_{\mathrm{VCyc}}$ is an isomorphism.

Remark 1.5. Farrell-Jones really only conjectured this for $R=\mathbb{Z}$. Moreover, they wrote (in 1993) that they regard this and related conjectures only as estimates which best fit the known data at this time. It still fits all known data today.

For arbitrary rings the conjecture was formulated in [2]. The proofs discussed in this article all work for arbitrary rings and it seems unlikely that the conjecture holds for $R=\mathbb{Z}$ and all groups, but not for arbitrary rings. 
Remark 1.6. Let $\mathcal{F}$ be a family of subgroups of $G$. If $R$ is a ring such that $K_{*} R[F]=$ 0 for all $F \in \mathcal{F}$, then $H_{*}^{G}\left(E_{\mathcal{F}} G ; \mathbf{K}_{R}\right)=0$.

In particular, the Farrell-Jones Conjecture predicts the following: if $R$ is a ring such that $K_{*}(R[V])=0$ for all $V \in$ VCyc then $K_{*}(R[G])=0$ for all groups $G$.

Transitivity principle. The family in the Farrell-Jones Conjecture is fixed to be the family of virtually cyclic groups. Nevertheless, it is beneficial to keep the family flexible, because of the following transitivity principle [25, A.10].

Proposition 1.7. Let $\mathcal{F} \subseteq \mathcal{H}$ be families of subgroups of $G$. Write $\mathcal{F} \cap H$ for the family of subgroups of $H$ that belong to $\mathcal{F}$. Assume that

(a) $\alpha_{\mathcal{H}}: H_{*}^{G}\left(E_{\mathcal{H}} G ; \mathbf{K}_{R}\right) \rightarrow K_{*}(R[G])$ is an isomorphism,

(b) $\alpha_{\mathcal{F} \cap H}: H_{*}^{H}\left(E_{\mathcal{F} \cap H} H ; \mathbf{K}_{R}\right) \rightarrow K_{*}(R[H])$ is an isomorphism for all $H \in \mathcal{H}$.

Then $\alpha_{\mathcal{F}}: H_{*}^{G}\left(E_{\mathcal{F}} G ; \mathbf{K}_{R}\right) \rightarrow K_{*}(R[G])$ is an isomorphism.

Remark 1.8. The following illustrates the transitivity principle.

Assume that $R$ is a ring such that $K_{*}(R[F])=0$ for all $F \in \mathcal{F}$. Assume moreover that the assumptions of Proposition 1.7 are satisfied. Combining Remark 1.6 with (b) we conclude $K_{*}(R[H])=0$ for all $H \in \mathcal{H}$. Then combining Remark 1.6 with (a) it follows that $K_{*}(R[G])=0$.

Remark 1.9. The transitivity principle can be used to prove the Farrell-Jones Conjecture for certain classes by induction. For example the proof of the Farrell-Jones Conjecture for $\mathrm{GL}_{n}(\mathbb{Z})$ uses an induction on $n[11$. Of course the hard part is still to prove in the induction step that $\alpha_{\mathcal{F}_{n-1}}$ is an isomorphism for $\mathrm{GL}_{n}(\mathbb{Z})$ where the family $\mathcal{F}_{n-1}$ contains only groups that can be build from $\mathrm{GL}_{n-1}(\mathbb{Z})$ and poly-cyclic groups. The induction step uses Theorem B from Section 3 , See also Remark 5.21

More general coefficients. Farrell and Jones also introduced a generalization of their conjecture now called the fibered Farrell-Jones Conjecture. This version of the conjecture is often not harder to prove than the original conjecture. Its advantage is that it has better inheritance properties. An alternative to the fibered conjecture is to allow more general coefficients where the group can act on the ring. As $K$ theory only depends on the category of finitely generated projective modules and not on the ring itself, it is natural to also replace the ring by an additive category. We briefly recall this generalization from 13 .

Let $\mathcal{A}$ be an additive category with a $G$-action. There is a construction of an additive category $\mathcal{A}[G]$ that generalizes the twisted group ring for actions of $G$ on a $\operatorname{ring} R$. (In the notation of [13, Def. 2.1] this category is denoted as $\mathcal{A} *_{G} G / G ; \mathcal{A}[G]$ is a more descriptive name for it.) There is also a homology theory $H_{*}^{G}\left(-; \mathbf{K}_{\mathcal{A}}\right)$ for $G$-spaces such that $H_{*}^{G}\left(G / H ; \mathbf{K}_{\mathcal{A}}\right)=K_{*}(\mathcal{A}[H])$. Therefore there are assembly maps

$$
\alpha_{\mathcal{F}}: H_{*}^{G}\left(E_{\mathcal{F}} G ; \mathbf{K}_{\mathcal{A}}\right) \rightarrow H_{*}^{G}\left(G / G ; \mathbf{K}_{\mathcal{A}}\right)=K_{*}(\mathcal{A}[G]) .
$$

Conjecture 1.10 (Farrell-Jones Conjecture with coefficients). For all groups $G$ and all additive categories $\mathcal{A}$ with $G$-action the assembly map $\alpha_{\mathrm{VCyc}}$ is an isomorphism.

An advantage of this version of the conjecture is the following inheritance property.

Proposition 1.11. Let $N \longmapsto G \rightarrow Q$ be an extension of groups. Assume that $Q$ and all preimages of virtually cyclic subgroups under $G \rightarrow Q$ satisfies the FarrellJones Conjecture with coefficients 1.10. Then $G$ satisfies the Farrell-Jones Conjecture with coefficients 1.10 . 
Remark 1.12. Proposition 1.11 can be used to prove the Farrell-Jones Conjecture with coefficients for virtually nilpotent groups using the conjecture for virtually abelian groups, compare [10, Thm. 3.2].

It can also be used to reduce the conjecture for virtually poly-cyclic groups to irreducible special affine groups [3, Sec. 4]. The latter class consists of certain groups $G$ for which there is an exact sequence $\Delta \rightarrow G \rightarrow D$, where $D$ is infinite cyclic or the infinite dihedral group and $\Delta$ is a crystallographic group.

Remark 1.13. For additive categories with $G$-action the consequence from Remark 1.6 becomes an equivalent formulation of the conjecture: A group $G$ satisfies the Farrell-Jones Conjecture with coefficients [1.10 if and only if for additive categories $\mathcal{B}$ with $G$-action we have

$$
K_{*}(\mathcal{B}[V])=0 \text { for all } V \in \mathrm{VCyc} \Longrightarrow K_{*}(\mathcal{B}[G])=0 .
$$

(This follows from [9, Prop. 3.8] because the obstruction category $\mathcal{O}^{G}\left(E_{\mathcal{F}} G ; \mathcal{A}\right.$ ) is equivalent to $\mathcal{B}[G]$ for some $\mathcal{B}$ with $K_{*}(\mathcal{B}[F])=0$ for all $F \in \mathcal{F}$.)

In particular, surjectivity implies bijectivity for the Farrell-Jones Conjecture with coefficients.

Remark 1.14. The Farrell-Jones Conjecture 1.4 should be viewed as a conjecture about finitely generated groups. If it holds for all finitely generated subgroups of a group $G$, then it holds for $G$. The reason for this is that the conjecture is stable under directed unions of groups [27, Thm. 7.1].

With coefficients the situation is even better. This version of the conjecture is stable under directed colimits of groups [4, Cor. 0.8]. Consequently the FarrellJones Conjecture with coefficients holds for all groups if and only if it holds for all finitely presented groups, compare [1, Cor. 4.7]. It is therefore a conjecture about finitely presented groups.

Despite the usefulness of this more general version of the conjecture I will mostly ignore it in this paper to keep the notation a little simpler.

$L$-theory. There is a version of the Farrell-Jones Conjecture for $L$-theory. For some applications this is very important. For example the Borel Conjecture asserting the rigidity of closed aspherical topological manifolds follows in dimensions $\geq 5$ via surgery theory from the Farrell-Jones Conjecture in $K$ - and $L$-theory. The $L$-theory version of the conjecture is very similar to the $K$-theory version. Everything said so far about the $K$-theory version also holds for the $L$-theory version.

For some time proofs of the $L$-theoretic Farrell-Jones conjecture have been considerably harder than their $K$-theoretic analoga. Geometric transfer arguments used in $L$-theory are considerably more involved than their counterparts in $K$ theory. A change that came with considering arbitrary rings as coefficients in [2, is that transfers became more algebraic. It turned out 6 that this more algebraic point of view allowed for much easier $L$-theory transfers. (In essence, because the world of chain complexes with Poincaré duality is much more flexible than the world of manifolds.) This is elaborated at the end of Section 4.

I think that it is fair to say that, as far as proofs are concerned, there is as at the moment no significant difference between the $K$-theoretic and the $L$-theoretic Farrell-Jones Conjecture. For this reason $L$-theory is not discussed in much detail in these notes.

\section{Controlled topology}

The thin $h$-cobordism theorem. An $h$-cobordism $W$ is a compact manifold whose boundary is a disjoint union $\partial W=\partial_{0} W \amalg \partial_{1} W$ of closed manifolds such that the inclusions $\partial_{0} W \rightarrow W$ and $\partial_{1} W \rightarrow W$ are homotopy equivalences. If 
$M=\partial_{0} W$, then we say $W$ is an $h$-cobordism over $M$. If $W$ is homeomorphic to $M \times[0,1]$, then $W$ is called trivial.

Definition 2.1. Let $M$ be a closed manifold with a metric $d$. Let $\varepsilon \geq 0$.

An $h$-cobordism $W$ over $M$ is said to be $\varepsilon$-controlled over $M$ if there exists a retraction $p: W \rightarrow M$ for the inclusion $M \rightarrow W$ and a homotopy $H: \operatorname{id}_{W} \rightarrow p$ such that for all $x \in W$ the track

$$
\{p(H(t, x)) \mid t \in[0,1]\} \subseteq M
$$

has diameter at most $\varepsilon$.

Remark 2.2. Clearly, the trivial $h$-cobordism is 0 -controlled. Thus it is natural to think of being $\varepsilon$-controlled for small $\varepsilon$ as being close to the trivial $h$-cobordism.

The following theorem is due to Quinn [39, Thm. 2.7]. See [18, 19, 28, for closely related results by Chapman and Ferry.

Theorem 2.3 (Thin $h$-cobordism theorem). Assume $\operatorname{dim} M \geq 5$. Fix a metric $d$ on $M$ (generating the topology of $M$ ).

Then there is $\varepsilon>0$ such that all $\varepsilon$-controlled $h$-cobordisms over $M$ are trivial.

Remark 2.4. Farrell-Jones used the thin $h$-cobordism Theorem 2.3 and generalizations thereof to study $K_{*}(\mathbb{Z}[G]), * \leq 1$. For example in 23 they used the geodesic flow of a negatively curved manifold $M$ to show that any element in $\operatorname{Wh}\left(\pi_{1} M\right)$ could be realized by an $h$-cobordism that in turn had to be trivial by an application of (a generalization of) the thin $h$-cobordism Theorem. Thus $\mathrm{Wh}\left(\pi_{1} M\right)=0$. In later papers they replaced the thin $h$-cobordism theorem by controlled surgery theory and controlled pseudoisotopy theory.

The later proofs of the Farrell-Jones Conjecture that we discuss here do not depend on the thin $h$-cobordism Theorem, controlled surgery theory or controlled pseudoisotopy theory, but on a more algebraic control theory that we discuss in the next subsection.

An algebraic analog of the thin $h$-cobordism theorem. Geometric groups (later also called geometric modules) were introduced by Connell-Hollingsworth [20]. The theory was developed much further by, among others, Quinn and Pedersen and is sometimes referred to as controlled algebra. A very pleasant introduction to this theory is given in 37.

Let $R$ be a ring and $G$ be a group.

Definition 2.5. Let $X$ be a free $G$-space and $p: X \rightarrow Z$ be a $G$-map to a metric space with an isometric $G$-action.

(a) A geometric $R[G]$-module over $X$ is a collection $\left(M_{x}\right)_{x \in X}$ of finitely generated free $R$-modules such that the following two conditions are satisfied.

- $M_{x}=M_{g x}$ for all $x \in X, g \in G$.

$-\left\{x \in X \mid M_{x} \neq 0\right\}=G \cdot S_{0}$ for some finite subset $S_{0}$ of $X$.

(b) Let $M$ and $N$ be geometric $R[G]$-modules over $X$. Let $f: \bigoplus_{x \in X} M_{x} \rightarrow$ $\bigoplus_{x \in X} N_{x}$ be an $R[G]$-linear map (for the obvious $R[G]$-module structures). Write $f_{x^{\prime \prime}, x^{\prime}}$ for the composition

$$
M_{x^{\prime}} \longmapsto \bigoplus_{x \in X} M_{x} \stackrel{f}{\rightarrow} \bigoplus_{x \in X} N_{x} \rightarrow N_{x^{\prime \prime}}
$$

The support of $f$ is defined as supp $f:=\left\{\left(x^{\prime \prime}, x^{\prime}\right) \mid f_{x^{\prime \prime}, x^{\prime}} \neq 0\right\} \subseteq X \times X$. Let $\varepsilon \geq 0$. Then $f$ is said to be $\varepsilon$-controlled over $Z$ if

$$
d_{Z}\left(p\left(x^{\prime \prime}\right), p\left(x^{\prime}\right)\right) \leq \varepsilon \quad \text { for all } \quad\left(x^{\prime \prime}, x^{\prime}\right) \in \operatorname{supp} f .
$$


(c) Let $M$ be a geometric $R[G]$-module over $X$. Let $f$ : $\bigoplus_{x \in X} M_{x} \rightarrow \bigoplus_{x \in X} M_{x}$ be an $R[G]$-automorphism. Then $f$ is said to be an $\varepsilon$-automorphism over $Z$ if both $f$ and $f^{-1}$ are $\varepsilon$-controlled over $Z$.

Remark 2.6. Geometric $R[G]$-modules over $X$ are finitely generated free $R[G]$ modules with an additional structure, namely an $G$-equivariant decomposition into $R$-modules indexed by points in $X$. This additional structure is not used to change the notion of morphisms which are still $R[G]$-linear maps. But this structure provides an additional point of view for $R[G]$-linear maps: the set of morphisms between two geometric $R[G]$-modules now carries a filtration by control.

A good (and very simple) analog is the following. Consider finitely generated free $R$-modules. An additional structure one might be interested in are bases for such modules. This additional information allows us to view $R$-linear maps between them as matrices.

Controlled algebra is really not much more than working with (infinite) matrices whose index set is a (metric) space. Nevertheless this theory is very useful and flexible.

It is a central theme in controlled topology that sufficiently controlled obstructions (for example Whitehead torsion) are trivial. Another related theme is that assembly maps can be constructed as forget-control maps. In this paper we will use a variation of this theme for $K_{1}$ of group rings over arbitrary rings. Before we can state it we briefly fix some conventions for simplicial complexes.

Convention 2.7. Let $\mathcal{F}$ be a family of subgroups of $G$. By a simplicial $(G, \mathcal{F})$ complex we shall mean a simplicial complex $E$ with a simplicial $G$-action whose isotropy groups $G_{x}=\{g \in G \mid g \cdot x=x\}$ belong to $\mathcal{F}$ for all $x \in E$.

Convention 2.8. We will always use the $l^{1}$-metric on simplicial complexes. Let $Z^{(0)}$ be the vertex set of the simplicial complex $Z$. Then every element $z \in Z$ can be uniquely written as $z=\sum_{v \in Z^{(0)}} z_{v} \cdot v$ where $z_{v} \in[0,1]$, all but finitely many $z_{v}$ are zero and $\sum_{v \in Z^{(0)}} z_{v}=1$. The $l^{1}$-metric on $Z$ is given by

$$
d_{Z}^{1}\left(z, z^{\prime}\right)=\sum_{v \in V}\left|z_{v}-z^{\prime}{ }_{v}\right|
$$

Remark 2.9. If $E$ is a simplicial complex with a simplicial $G$-action such that the isotropy groups $G_{v}$ belong to $\mathcal{F}$ for all vertices $v \in E^{(0)}$ of $E$, then $E$ is a simplicial $(G, V \mathcal{F})$-complex, where $V \mathcal{F}$ consists of all subgroups $H$ of $G$ that admit a subgroup of finite index that belongs to $\mathcal{F}$.

Theorem 2.10 (Algebraic thin $h$-cobordism theorem). Given a natural number $N$, there is $\varepsilon_{N}>0$ such that the following holds: Let

(a) $Z$ be a simplicial $(G, \mathcal{F})$-complex of dimension at most $N$,

(b) $p: X \rightarrow Z$ be a $G$-map, where $X$ is a free $G$-space,

(c) $M$ be a geometric $R[G]$-module over $X$,

(d) $f: M \rightarrow M$ be an $\varepsilon_{N}$-automorphism over $Z$ (with respect to the $l^{1}$-metric on $Z$ ).

Then the $K_{1}$-class $[f]$ of $f$ belongs to the image of the assembly map

$$
\alpha_{\mathcal{F}}: H_{1}^{G}\left(E_{\mathcal{F}} G ; \mathbf{K}_{R}\right) \rightarrow K_{1}(R[G]) .
$$

Remark 2.11. I called Theorem 2.10 the algebraic thin $h$-cobordism theorem here, because it can be used to prove the thin $h$-cobordism theorem. Very roughly, this works as follows. Let $W$ be an $\varepsilon$-thin $h$-cobordism over $M$. Let $G=\pi_{1} M=$ $\pi_{1} W$. The Whitehead torsion of $W$ can be constructed using the singular chain complexes of the universal covers $\widetilde{W}$ and $\widetilde{M}$. This realizes the Whitehead torsion 
$\tau_{W} \in \mathrm{Wh}(G)$ of $W$ by an $\widetilde{\varepsilon}$-automorphism $f_{W}$ over $\widetilde{M}$, i.e. $\left[f_{W}\right]$ maps to $\tau_{W}$ under $K_{1}(\mathbb{Z}[G]) \rightarrow \mathrm{Wh}(G)$. Moreover, $\widetilde{\varepsilon}$ can be explicitly bounded in terms of $\varepsilon$, such that $\widetilde{\varepsilon} \rightarrow 0$ as $\varepsilon \rightarrow 0$. Because $\widetilde{M}$ is a free $G=\pi_{1} M$-space it follows from Theorem 2.10 that $\left[f_{W}\right]$ belongs to the image of the assembly map $\alpha: H_{1}^{G}\left(E G, \mathbf{K}_{\mathbb{Z}}\right) \rightarrow K_{1}(\mathbb{Z}[G])$. But $\mathrm{Wh}(G)$ is the cokernel of $\alpha$ and therefore $\tau_{W}=0$. This reduces the thin $h$-cobordism theorem to the $s$-cobordism theorem.

I believe that - at least in spirit - this outline is very close to Quinn's proof in 39 .

Remark 2.12. If $f: M \rightarrow M^{\prime}$ is $\varepsilon$-controlled over $Z$ and and $f^{\prime}: M^{\prime} \rightarrow M^{\prime \prime}$ is $\varepsilon^{\prime}$ controlled over $Z$, then their composition $f^{\prime} \circ f$ is $\varepsilon+\varepsilon^{\prime}$-controlled. In particular, there is no category whose objects are geometric modules and whose morphisms are $\varepsilon$-controlled for fixed (small) $\varepsilon$. However, there are very elegant substitutes for this ill-defined category. These are built by considering a variant of the theory over an open cone over $Z$ and taking a quotient category. In this quotient category every morphisms has for every $\varepsilon>0$ an $\varepsilon$-controlled representative. Pedersen-Weibel 38 ] used this to construct homology of a space $E$ with coefficients in the $K$-theory spectrum as the $K$-theory of an additive category. Similar constructions can be used to describe the assembly maps as forget-control maps 2, 17. This also leads to a category (called the obstruction category in [9]), whose $K$-theory describes the fiber of the assembly map. A minor drawback of these constructions is that they usually involve a dimension shift.

A very simple version of such a construction is discussed at the end of this section. See in particular Theorem 2.20.

Remark 2.13. It is not hard to deduce Theorem 2.10 from [6, Thm. 5.3]. The latter result is a corresponding result for the obstruction category mentioned in Remark 2.12 In fact this result about the obstruction is stronger and can be used to prove that the assembly map is an isomorphism and not just surjective, see 6 , Thm. 5.2]. I have elected to state the weaker Theorem 2.10 because it is much easier to state, but still grasps the heart of the matter. On the other hand, I think it is not at all easier to prove Theorem 2.10 than to prove the corresponding statement for the obstruction category. (The result in [6] deals with chain complexes, but this is not an essential difference.)

Remark 2.14. Results like Theorem 2.10 are very useful to prove the Farrell-Jones Conjecture. But it is not clear to me, that it really provides the best possible description of the image of the assembly map. For $g \in G$ we know that $[g]$ lies in the image of the assembly map. But its most natural representative (namely the isomorphism of $R[G]$ given by right multiplication by $g$ ) is not $\varepsilon$-controlled for small $\varepsilon$.

It may be beneficial to find other, maybe more algebraic and less geometric, characterizations of the image of the assembly map. But I do not know how to approach this.

Remark 2.15. The use of the $l^{1}$-metric in Theorem 2.10 is of no particular importance. In order for $\varepsilon_{N}$ to only depend on $N$ and not on $Z$, one has to commit to some canonical metric.

Remark 2.16. If $\mathcal{F}$ is closed under finite index supergroups, i.e., if $\mathcal{F}=V \mathcal{F}$ then there is no loss of generality in assuming that $Z$ is the $N$-skeleton of the model for $E_{\mathcal{F}} G$ discussed in Example 1.2. This holds because there is always a $G$-map $Z^{(0)} \rightarrow S:=\coprod_{F \in \mathcal{F}} G / F$ and this map extends to a simplicial map $Z \rightarrow \Delta(S)^{(N)}$. Barycentric subdivision only changes the metric on the $N$-skeleton in a controlled (depending on $N$ ) way. 
Remark 2.17. There is also a converse to Theorem 2.10, If $a \in K_{1}(R[G])$ lies in the image of the assembly map $\alpha_{\mathcal{F}}$ then there is some $N$ such that it can for any $\varepsilon>0$ be realized by an $\varepsilon$-automorphism over an $N$-dimensional simplicial complex $Z$ with a simplicial $G$-action all whose isotropy groups belong to $\mathcal{F}$. The simplicial complex can be taken to be the $N$-skeleton of a simplicial complex model for $E_{\mathcal{F}} G$.

This is a consequence of the description of the assembly map as a forget-control map as for example in [2, Cor. 6.3].

Remark 2.18. It is not hard to extend the theory of geometric $R[G]$-modules from rings to additive categories. In this case one considers collections $\left(A_{x}\right)_{x \in X}$ where each $A_{x}$ is an object from $\mathcal{A}$. In fact [6, Thm. 5.3], which implies Theorem 2.10, is formulated using additive categories as coefficients.

Remark 2.19. Results for $K_{1}$ often imply results for $K_{i}, i \leq 0$, using suspension rings. For a ring $R$, there is a suspension ring $\Sigma R$ with the property that $K_{i}(R)=$ $K_{i+1}(\Sigma R)$ [4]. This construction can be arranged to be compatible with group rings: $\Sigma(R[G])=(\Sigma R)[G]$. A consequence of this is that for a fixed group $G$ the surjectivity of $\alpha_{\mathcal{F}}: H_{1}^{G}\left(E_{\mathcal{F}} G ; \mathbf{K}_{R}\right) \rightarrow K_{1}(R[G])$ for all rings $R$ implies the surjectivity of $\alpha_{\mathcal{F}}$ for all $i \leq 1$, compare [2, Cor. 7.3].

Because of this trick there is no need for a version of Theorem 2.10 for $K_{i}, i \leq 0$.

Higher $K$-theory. We end this section by a brief discussion of a version of Theorem 2.10 for higher $K$-theory. Because there is no good concrete description of elements in higher $K$-theory it will use slightly more abstract language.

Let $p_{n}: X_{n} \rightarrow Z_{n}$ be a sequence of $G$-maps where each $X_{n}$ is a free $G$-space and each $Z_{n}$ is a simplicial $(G, \mathcal{F})$-complex of dimension $N$. Define a category $\mathcal{C}$ as follows. Objects of $\mathcal{C}$ are sequences $\left(M_{n}\right)_{n \in \mathbb{N}}$ where for each $n, M_{n}$ is a geometric $R[G]$-module over $X_{n}$. A morphism $\left(M_{n}\right)_{n \in \mathbb{N}} \rightarrow\left(N_{n}\right)_{n \in \mathbb{N}}$ in $\mathcal{C}$ is given by a sequence $\left(f_{n}\right)_{n \in \mathbb{N}}$ of $R[G]$-linear maps $f_{n}: \bigoplus_{x \in X_{n}}\left(M_{n}\right)_{x} \rightarrow \bigoplus_{x \in X_{n}}\left(N_{n}\right)_{x}$ satisfying the following condition: there is $\alpha>0$ such that for each $n, f_{n}$ is $\frac{\alpha}{n}$ controlled over $Z_{n}$. For each $k \in \mathbb{N}$,

$$
\left(M_{n}\right)_{n \in \mathbb{N}} \mapsto \bigoplus_{x \in X_{k}}\left(M_{k}\right)_{x}
$$

defines a functor $\pi_{k}$ from $\mathcal{C}$ to the category of finitely generated free $R[G]$-modules. The following is a variation of [14, Cor. 4.3]. It can be proven using [9, Thm. 7.2].

Theorem 2.20. Let $a \in K_{*}(R[G])$. Suppose that there is $A \in K_{*}(\mathcal{C})$ such that for all $k$

$$
\left(\pi_{k}\right)_{*}(A)=a .
$$

Then a belongs to the image of $\alpha_{\mathcal{F}}: H_{*}^{G}\left(E_{\mathcal{F}} G ; \mathbf{K}_{R}\right) \rightarrow K_{*}(R[G])$.

\section{Conditions that imply the Farrell-Jones Conjecture}

In [6, 9] the Farrell-Jones Conjecture is proven for hyperbolic and CAT(0)-groups. Both papers take a somewhat axiomatic point of view. They both contain careful (and somewhat lengthy) descriptions of conditions on groups that imply the FarrellJones conjecture. The conditions in the two papers are closely related to each other. A group satisfying them is said to be transfer reducible over a given family of subgroups in 6]. Further variants of these conditions are introduced in [11, 45. The existence of all these different versions of these conditions seem to me to suggest that we have not found the ideal formulation of them yet. The notion of transfer reducible groups (and all its variations) can be viewed as an axiomatization of the work of Farrell-Jones using the geodesic flow that began with 23. Somewhat different conditions - related to work of Farrell-Hsiang [22] - are discussed in [5]. 
Transfer reducible groups - strict version. Let $R$ be a ring and $G$ be a group.

Definition 3.1. An $N$-transfer space $X$ is a compact contractible metric space such that the following holds.

For any $\delta>0$ there exists a simplicial complex $K$ of dimension at most $N$ and continuous maps and homotopies $i: X \rightarrow K, p: K \rightarrow X$, and $H: p \circ i \rightarrow \operatorname{id}_{X}$ such that for any $x \in X$ the diameter of $\{H(t, x) \mid t \in[0,1]\}$ is at most $\delta$.

Example 3.2. Let $T$ be a locally finite simplicial tree. The compactification $\bar{T}$ of $T$ by equivalence classes of geodesic rays is a 1-transfer space.

Theorem A. Suppose that $G$ is finitely generated by $S$. Let $\mathcal{F}$ be a family of subgroups of $G$. Assume that there is $N \in \mathbb{N}$ such that for any $\varepsilon>0$ there are

(a) an $N$-transfer space $X$ equipped with a $G$-action,

(b) a simplicial $(G, \mathcal{F})$-complex $E$ of dimension at most $N$,

(c) a map $f: X \rightarrow E$ that is $G$-equivariant up to $\varepsilon: d^{1}(f(s \cdot x), s \cdot f(x)) \leq \varepsilon$ for all $s \in S, x \in X$.

Then $\alpha_{\mathcal{F}}: H_{*}^{G}\left(E_{\mathcal{F}} G ; \mathbf{K}_{R}\right) \rightarrow K_{*}(R G)$ is an isomorphism.

Remark 3.3. It follows from 8 that Theorem $\mathrm{A}$ (with $\mathcal{F}$ the family of virtually cyclic subgroups $\mathrm{VCyc})$ applies to hyperbolic groups.

Example 3.4. Let $G$ be a group and $K$ be a finite contractible simplicial complex with a simplicial $G$-action. Then for the family $\mathcal{F}:=\mathcal{F}_{K}$ the assembly map $\alpha_{\mathcal{F}}: H_{*}^{G}\left(E_{\mathcal{F}} G ; \mathbf{K}_{R}\right) \rightarrow K_{*}(R G)$ is an isomorphism. This follows from Theorem $\mathrm{A}$ by setting $N:=\operatorname{dim} K$ and $X:=K, E:=K, f:=\operatorname{id}_{K}$ (for all $\varepsilon>0$ ). Since $K$ is finite, the group of simplicial automorphisms of $K$ is also finite. It follow that for all $x \in K$ the isotropy group $G_{x}$ has finite index in $G$.

The assumptions of Theorem $\mathrm{A}$ should be viewed as a weakening of this example. The properties of $K$ are reflected in requirements on $X$ or on $E$ and the existence of the map $f$ yields a strong relationship between $X$ and $E$.

Remark 3.5. Rufus Willet and Guoliang Yu pointed out that the assumption of Theorem $\mathrm{A}$ implies that the group $G$ has finite asymptotic dimension, provided there is a uniform bound on the asymptotic dimension of groups in $\mathcal{F}$. The latter assumptions is of course satisfied for the family of virtually cyclic groups VCyc.

Remark 3.6. Martin Bridson pointed out that the assumptions of Theorem $\mathrm{A}$ are formally very similar to the concept of amenability for actions on compact spaces. The main difference is that in the latter context $E$ is replaced by the (infinite dimensional) space of probability measures on $G$.

Remark 3.7. Theorem $\mathrm{A}$ is a minor reformulation of [9, Thm. 1.1]. In this reference instead of the existence of $f$ the existence of certain covers $\mathcal{U}$ of $G \times X$ are postulated. But the first step in the proof is to use a partition of unity to construct a $G$-map from $G \times X$ to the nerve $|\mathcal{U}|$ of $\mathcal{U}$. Under the assumptions formulated in Theorem $\mathrm{A}$ this map is simply $(g, x) \mapsto g \cdot f\left(g^{-1} x\right)$.

Avoiding the open covers makes the theorem easier to state, but there is no real mathematical difference.

Remark 3.8. The proof of Theorem $\mathrm{A}$ in 9 really shows a little bit more: there is $M$ (depending on $N$ ) such that the restriction of $\alpha_{\mathcal{F}}$ to $H_{*}^{G}\left(E_{\mathcal{F}} G^{(M)} ; \mathbf{K}_{R}\right)$ is surjective. For arbitrary groups and rings with non-trivial $K$-theory in infinitely many negative degrees there will be no such $M$. It is reasonable to expect that groups satisfying the assumptions of Theorem $\mathrm{A}$ will also admit a finite dimensional model for the space $E_{\mathcal{F}} G$. 
Remark 3.9. Let $E$ be a simplicial complex of dimension $N$. Let $g$ be a simplicial automorphism of $E$. Let $x=\sum_{v \in E^{(0)}} x_{v} \cdot v$ be a point of $E$. Let $\operatorname{supp} x:=\{v \in$ $\left.E^{(0)} \mid x_{v} \neq 0\right\}$. It is a disjoint union of the sets

$$
\begin{aligned}
P_{x} & :=\left\{v \in \operatorname{supp} x \mid \forall n \in \mathbb{N}: g^{n} \in \operatorname{supp} x\right\} \\
D_{x} & :=\left\{v \in \operatorname{supp} x \mid \exists n \in \mathbb{N}: g^{n} \notin \operatorname{supp} x\right\} .
\end{aligned}
$$

Observe that for $v \in D_{x}$, we have $d^{1}(x, g x) \geq x_{v}$. Assume now that $d^{1}(x, g x)<$ $\frac{1}{N+1}$. As $\sum_{v} x_{v}=1$ there is a vertex $v$ with $v \geq \frac{1}{N+1}$. Such a vertex $v$ belongs then to $P_{x}$ and it follows that $\left\{g^{n} v \mid n \in \mathbb{N}\right\}$ is finite and spans a simplex of $E$ whose barycenter is fixed by $g$.

Assume now that $f: X \rightarrow E$ is as in assumption (c) of Theorem A. If $G_{x}$ is the isotropy group for $x \in X$ (and if $G_{x}$ is finitely generated by $S_{x}$ say) then if $\varepsilon$ is sufficiently small it follows that $d^{1}(f(x), g f(x))<\frac{1}{N+1}$. The previous observation implies then $G_{x} \in \mathcal{F}$.

On the other hand one can apply the Lefschetz fixed point theorem to the simplicial dominations to $X$ and finds for fixed $g \in G$ and each $\varepsilon>0$ a point $x_{\varepsilon} \in X$ such that $d\left(g x_{\varepsilon}, x_{\varepsilon}\right) \leq \varepsilon$. The compactness of $X$ implies that there is a fixed point in $X$ for each element of $G$. Altogether, it follows that $\mathcal{F}$ will necessarily contain the family of cyclic subgroups.

Remark 3.10. Frank Quinn has shown that one can replace the family of virtually cyclic groups in the Farrell-Jones Conjecture by the family of (possibly infinite) hyper-elementary groups 40 .

It is an interesting question whether one can (maybe using Smith theory) build on the argument from Remark 3.9 to conclude that in order for the assumptions of Theorem A to be satisfied it is necessary for $\mathcal{F}$ to contain this family of (possibly infinite) hyper-elementary groups.

Remark 3.11. One can ask for which $N$-transfer spaces $X$ with a $G$-action it is possible to find for all $\varepsilon>0$ a map $f: X \rightarrow E$ as in assumptions (b)] and (c)

Remark 3.9 shows that a necessary condition is $G_{x} \in \mathcal{F}$ for all $x \in X$, but it is not clear to me that this condition is not sufficient.

In light of the observation of Willet and Yu from Remark 3.5 a related question is whether there is a group $G$ of infinite asymptotic dimension for which there is an $N$-transfer space with a $G$-action such that the asymptotic dimension of $G_{x}, x \in X$ is uniformly bounded.

Remark 3.12. The reader is encouraged to try to check that finitely generated free groups satisfy the assumptions of Theorem A with respect to the family of (virtually) cyclic subgroups. In this case one can use the compactification $\bar{T}$ of the usual tree by equivalence classes of geodesic rays as the transfer space. I am keen to see a proof of this that is easier than the one coming out of [8] and avoids flow spaces. Maybe a clever application of Zorn's Lemma could be useful here.

I am not completely sure whether or not it is possible to write down the maps $f: \bar{T} \rightarrow E$ in assumption (c) explicitly for finitely generated free groups.

Transfer reducible groups - homotopy version. Let $R$ be a ring.

Definition 3.13. Let $G=\langle S \mid R\rangle$ be a finitely presented group. A homotopy action of $G$ on a space $X$ is given by

- for all $s \in S \cup S^{-1}$ maps $\varphi_{s}: X \rightarrow X$,

- for all $r=s_{1} \cdot s_{2} \cdots s_{l} \in R$ homotopies $H_{r}: \varphi_{s_{1}} \circ \varphi_{s_{2}} \circ \cdots \circ \varphi_{s_{l}} \rightarrow \mathrm{id}_{X}$

Theorem B. Suppose that $G=\langle S \mid R\rangle$ is a finitely presented group. Let $\mathcal{F}$ be a family of subgroups of $G$. Assume that there is $N \in \mathbb{N}$ such that for any $\varepsilon>0$ there are 
(a) an $N$-transfer space $X$ equipped with a homotopy $G$-action $(\varphi, H)$,

(b) a simplicial $(G, \mathcal{F})$-complex $E$ of dimension at most $N$,

(c) a map $f: X \rightarrow E$ that is $G$-equivariant up to $\varepsilon$ : for all $x \in X, s \in S \cup S^{-1}$, $r \in R$

$-d^{1}\left(f\left(\varphi_{s}(x)\right), s \cdot f(x)\right) \leq \varepsilon$,

- $\left\{H_{r}(t, x) \mid t \in[0,1]\right\}$ has diameter at most $\varepsilon$.

Then $\alpha_{\mathcal{F}}: H_{i}^{G}\left(E_{\mathcal{F}} G ; \mathbf{K}_{R}\right) \rightarrow K_{i}(R G)$ is an isomorphism for $i \leq 0$ and surjective for $i=1$.

Remark 3.14. It follows from [7] that Theorem B] applies to CAT(0)-groups (where $\mathcal{F}$ is the family of virtually cyclic groups). We will sketch the proof of this fact in Section 5 .

Wegner introduced the notion of a strong homotopy action and proved a version of Theorem $\mathrm{B}$ where the conclusion is that $\alpha_{\mathcal{F}}$ is an isomorphism in all degrees [45]. This result also applies to CAT(0)-groups.

Remark 3.15. Theorem $\mathrm{B}$ is a reformulation of [6, Thm. 1.1] (just as in Remark 3.7).

The assumptions of Theorem $\mathrm{A}$ feel much cleaner than the assumptions of Theorem $\mathrm{B}$. It would be very interesting if one could show, maybe using some kind of limit that promotes a (strong) homotopy action to an actual action, such that the latter (or Wegner's variation of them) do imply the former.

In light of Remark 3.5 this would imply in particular that CAT(0)-groups have finite asymptotic dimension and is therefore probably a difficult (or impossible) task.

Remark 3.16. I do not know whether semi-direct products of the form $\mathbb{Z}^{n} \rtimes \mathbb{Z}$ satisfy the assumptions of Theorem $\mathrm{B}$, for example if $\mathcal{F}$ is the family of abelian groups. On the other hand the Farrell-Jones Conjecture is known to hold for such groups and more general for virtually poly-cyclic groups [3].

Remark 3.17. Remark 3.8 also applies to Theorem B.

Remark 3.18. There is an $L$-theory version of Theorem B, see [6, Thm. 1.1(ii)]. There, the conclusion is that the assembly map $\alpha_{\mathcal{F}_{2}}$ is an isomorphism in $L$-theory where $\mathcal{F}_{2}$ is the family of subgroups that contain a member of $\mathcal{F}$ as a subgroup of index at most 2. Of course $\mathrm{VCyc}=\mathrm{VCyc}_{2}$. There is no restriction on the degree $i$ in this $L$-theoretic version and so this also provides an $L$-theory version of Theorem $\mathrm{A}$

\section{Farrell-Hsiang groups.}

Definition 3.19. A finite group $H$ is said to be hyper-elementary if there exists a short exact sequence

$$
C \longmapsto H \rightarrow P
$$

where $C$ is a cyclic group and the order of $P$ is a prime power.

Quinn generalized this definition to infinite groups by allowing the cyclic group to be infinite 40.

Hyper-elementary groups play a special role in $K$-theory because of the following result of Swan 43 . For a group $G$ we denote by $\operatorname{Sw}(G)$ the $\operatorname{Swan}$ group of $G$. It can be defined as $K_{0}$ of the exact category of $\mathbb{Z}[G]$-modules that are finitely generated free as $\mathbb{Z}$-modules. This group encodes information about transfer maps in algebraic $K$-theory.

Theorem 3.20 (Swan). For a finite group $F$ the induction maps combine to a surjective map

$$
\bigoplus_{H \in \mathcal{H}(F)} \mathrm{Sw}(H) \rightarrow \mathrm{Sw}(F)
$$


where $\mathcal{H}(F)$ denotes the family of hyper-elementary subgroups of $F$.

Let $R$ be a ring and $G$ be a group.

Theorem C. Suppose that $G$ is finitely generated by $S$. Assume that there is $N \in \mathbb{N}$ such that for any $\varepsilon>0$ there are

(a) a group homomorphism $\pi: G \rightarrow F$ where $F$ is finite,

(b) a simplicial $(G, \mathcal{F})$-complex $E$ of dimension at most $N$

(c) a map $f: \bigsqcup_{H \in \mathcal{H}(F)} G / \pi^{-1}(H) \rightarrow E$ that is $G$-equivariant up to $\varepsilon: d^{1}(f(s x)$, s. $f(x)) \leq \varepsilon$ for all $s \in S, x \in \coprod_{H \in \mathcal{H}(F)} G / \pi^{-1}(H)$.

Then $\alpha_{\mathcal{F}}: H_{*}^{G}\left(E_{\mathcal{F}} G ; \mathbf{K}_{R}\right) \rightarrow K_{*}(R G)$ is an isomorphism.

Remark 3.21. Theorem $[$ is proven in $[5$ building on work of Farrell-Hsiang 22 . The main difference to Theorems $\mathrm{A}$ and $\mathrm{B}$ is that the transfer space $X$ is replaced by the discrete space $\bigsqcup_{H \in \mathcal{H}(F)} G / \pi^{-1}(H)$. It is Swan's theorem 3.20 that replaces the contractibility of $X$.

I have no conceptual understanding of Swan's theorem. For this reason Theorem $[$ is to me not as conceptually satisfying as Theorem $\mathrm{A}$. Moreover, I expect that a version of Theorem $\mathrm{C}$ for Waldhausen's $A$-theory will need a larger family than the family of hyper-elementary subgroups.

Remark 3.22. Groups satisfying the assumption of Theorem C are called FarrellHsiang groups with respect to $\mathcal{F}$ in [5].

Remark 3.23. Theorem [C can be used to prove the Farrell-Jones Conjecture for virtually poly-cyclic groups [3, Sec. 3 and 4]. We will discuss some semi-direct products of the form $\mathbb{Z}^{n} \rtimes \mathbb{Z}$ in Section 6

Remark 3.24. Remark 3.8 also applies to Theorem C

Remark 3.25. Theorem $[$ holds without change in $L$-theory as well $[5$.

Remark 3.26. It would be good to find a natural common weakening of the assumptions in Theorems $\mathrm{A}[\mathrm{B}$ and $\mathrm{C}$ that still implies the Farrell-Jones Conjecture. Ideally such a formulation should have similar inheritance properties as the Farrell-Jones Conjecture, see Propositions 1.7 and 1.11 .

Injectivity. It is interesting to note that injectivity of the assembly map $\alpha_{\{1\}}$ or $\alpha_{\text {Fin }}$ is known for seemingly much bigger classes of groups, than the class of groups known to satisfy the Farrell-Jones Conjecture. Rational injectivity of the $L$-theoretic assembly map $\alpha_{\{1\}}$ is of particular interest, as it implies Novikov's conjecture on the homotopy invariance of higher signatures. Yu [46] proved the Novikov conjecture for all groups admitting a uniform embedding into a Hilbertspace. This class of groups contains all groups of finite asymptotic dimension. Integral injectivity of the assembly map $\alpha_{\{1\}}$ for $K$ - and $L$-theory is known for all groups that admit a finite $C W$-complex as a model for $B G$ and are of finite decomposition complexity [30, 41. The latter property is a generalization of finite asymptotic dimension. Rational injectivity of the $K$-theoretic assembly map $\alpha_{\{1\}}$ for the ring $\mathbb{Z}$ is proven by Bökstedt-Hsiang-Madsen [15] for all groups $G$ satisfying the following homological finiteness condition: for all $n$ the rational group-homology $H_{*}(G ; \mathbb{Q})$ is finite dimensional.

\section{On the Proof of Theorem A}

Using the results from controlled topology discussed in Section 2 we will outline a proof of the surjectivity of

$$
\alpha_{\mathcal{F}}: H_{1}^{G}\left(E_{\mathcal{F}} G ; \mathbf{K}_{R}\right) \rightarrow K_{1}(R[G])
$$

under the assumptions of Theorem $\mathrm{A}$. 
Step 1: preparations. Let $G$ be a finitely generated group and $\mathcal{F}$ be a family of subgroups of $G$. Let $N \in \mathbb{N}$ be the number appearing in Theorem A. Let $a \in K_{1}(R[G])$. Then $a=[\psi]$ where $\psi: R[G]^{n} \rightarrow R[G]^{n}$ is an $R[G]$-right linear automorphism. We write $R[G]^{n}=\mathbb{Z}[G] \otimes_{\mathbb{Z}} R^{n}$. There is a finite subset $T \subseteq G$ and there are $R$-linear maps $\psi_{g}: R^{n} \rightarrow R^{n}, \psi_{g}^{-1}: R^{n} \rightarrow R^{n}, g \in T$ such that

$$
\psi(h \otimes v)=\sum_{g \in T} h g^{-1} \otimes \psi_{g}(v) \quad \text { and } \quad \psi^{-1}(h \otimes v)=\sum_{g \in T} h g^{-1} \otimes \psi_{g}^{-1}(v) .
$$

Because of Theorem 2.10 it suffices to find

- a $G$-space $Y$,

- a $(G, \mathcal{F})$-complex $E$ of dimension at most $N$,

- a $G$-map $Y \rightarrow E$,

- a geometric $R[G]$-module $M$ over $Y$,

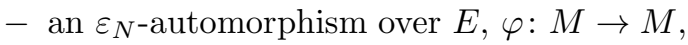

such that $[\varphi]=a \in K_{1}(R[G])$. Here $\varepsilon_{N}$ is the number depending on $N$, whose existence is asserted in Theorem 2.10 .

Let $L$ be a (large) number. We will later specify $L$; it will only depend on $N$. From the assumption of Theorem $\mathrm{A}$ we easily deduce that there are

(a) an $N$-transfer space $X$ equipped with a $G$-action,

(b) a simplicial $(G, \mathcal{F})$-complex $E$ of dimension at most $N$,

(c) a map $f: X \rightarrow E$ such that $d^{1}(f(g \cdot x), g \cdot f(x)) \leq \varepsilon_{N} / 2$ for all $x \in X$ and all $g \in G$ that can be written as $g=g_{1} \ldots g_{L}$ with $g_{1}, \ldots, g_{L} \in T$.

By compactness of $X$ there is $\delta_{0}>0$ such that $d^{1}\left(f(x), f\left(x^{\prime}\right)\right) \leq \varepsilon_{N} / 2$ for all $x, x^{\prime} \in X$ with $d\left(x, x^{\prime}\right) \leq L \delta_{0}$. We will use $Y:=G \times X$ with the $G$-action defined by $g \cdot(h, x):=(g h, x)$. We will also use the $G$-map $G \times X \rightarrow E,(g, x) \mapsto g f(x)$. The action of $G$ on $X$ will be used later.

Step 2: a chain complex over $X$. To simplify the discussion let us assume that for $X$ the maps $i$ and $p$ appearing in Definition 3.1 can be arranged to be $\delta$ homotopy equivalences. This means that in addition to $H$ there is also a homotopy $H^{\prime}: i \circ p \rightarrow \operatorname{id}_{K}$ such that for any $y \in K$ the diameter of $\left\{H^{\prime}(t, y) \mid t \in[0,1]\right\}$ with respect to the $l^{1}$-metric on $K$ is at most $\delta$.

Let $C_{*}$ be the simplicial chain complex of the $l$-fold simplicial subdivision of $K$. Using $p: K \rightarrow X$ we can view $C_{*}$ as a chain complex of geometric $\mathbb{Z}$-modules over $X$. If we choose $l$ sufficiently large, then we can arrange that the boundary maps $\partial^{C_{*}}$ of $C_{*}$ are $\delta_{0}$-controlled over $X$. Moreover, using the action of $G$ on $X$ and a $\delta$-homotopy equivalence between $K$ and $X$ (for $0<\delta \ll \delta_{0}$ ) and enlarging $l$ we can produce chain maps $\varphi_{g}: C_{*} \rightarrow C_{*}, g \in G$, chain homotopies $H_{g, h}: \varphi_{g} \circ \varphi_{h} \rightarrow \varphi_{g h}$ satisfying the following control conditions

- if $g \in T$ and $\left(x^{\prime}, x\right) \in \operatorname{supp} \varphi_{g}$ then $d\left(x^{\prime}, g x\right) \leq \delta_{0}$ (recall that we view $C_{*}$ as a chain complex over $X)$,

- if $g, h \in T$ and $\left(x^{\prime}, x\right) \in \operatorname{supp} H_{g, h}$ then $d\left(x^{\prime}, g h x\right) \leq \delta_{0}$.

Remark 4.1. If we drop the additional assumption on $X$ (i.e., if we no longer assume the existence of the homotopy $H^{\prime}$ ), then it is only possible to construct the chain complex $C_{*}$ in the idempotent completion of geometric $\mathbb{Z}$-modules over $X$. This is a technical but - I think - not very important point.

Remark 4.2. A construction very similar to this step 2 is carried out in great detail in [6, Sec. 8]. 
Step 3: transfer to a chain homotopy equivalence. Recall our automorphism $\psi$ of $R[G]^{n}=\mathbb{Z}[G] \otimes_{\mathbb{Z}} R^{n}$. We will now replace the $R$-module $R^{n}$ by the $R$-chain complex $C_{*} \otimes_{\mathbb{Z}} R^{n}$ to obtain the chain complex $D_{*}:=\mathbb{Z}[G] \otimes_{\mathbb{Z}} C_{*} \otimes_{\mathbb{Z}} R^{n}$. As $C_{*}$ is a chain complex of geometric $\mathbb{Z}$-modules over $X, D_{*}$ is naturally a geometric $R[G]$ module over $G \times X$. Here $\left(D_{*}\right)_{h, x}=\left\{h \otimes w \otimes v \mid v \in R^{n}, w \in\left(C_{*}\right)_{x}\right\}$ for $h \in G$, $x \in X$. Recall that we use the left action defined by $g \cdot(h, x)=(g h, x)$ on $G \times X$. We can now use the data from Step 2 to transfer $\psi$ to a chain homotopy equivalence $\Psi=\sum_{g \in T} g \otimes \varphi_{g} \otimes \psi_{g}: D_{*} \rightarrow D_{*}$. Similarly, there is a chain homotopy inverse $\Psi^{\prime}$ for $\Psi$ and there are chain homotopies $\mathcal{H}: \Psi \circ \Psi^{\prime} \rightarrow \operatorname{id}_{D_{*}}$ and $\mathcal{H}^{\prime}: \Psi^{\prime} \circ \Psi \rightarrow \operatorname{id}_{D_{*}}$. In more explicit formulas these are defined by

$$
\begin{aligned}
\Psi(h \otimes w \otimes v) & =\sum_{g \in T} h g^{-1} \otimes \varphi_{g}(w) \otimes \psi_{g}(v), \\
\Psi^{\prime}(h \otimes w \otimes v) & =\sum_{g \in T} h g^{-1} \otimes \varphi_{g}(w) \otimes \psi_{g}^{-1}(v), \\
\mathcal{H}(h \otimes w \otimes v) & =\sum_{g, g^{\prime} \in T} h\left(g g^{\prime}\right)^{-1} \otimes H_{g, g^{\prime}}(w) \otimes \psi_{g} \circ \psi_{g^{\prime}}^{-1}(v), \\
\mathcal{H}^{\prime}(h \otimes w \otimes v) & =\sum_{g, g^{\prime} \in T} h\left(g g^{\prime}\right)^{-1} \otimes H_{g, g^{\prime}}(w) \otimes \psi_{g}^{-1} \circ \psi_{g^{\prime}}(v),
\end{aligned}
$$

for $h \in G, w \in C_{*}, v \in R^{n}$.

Digression on torsion. Let $S$ be a ring. If $\Phi$ is a self-homotopy equivalence of a bounded chain complex $D_{*}$ of finitely generated free $S$-modules then its self-torsion $\tau(\Phi) \in K_{1}(S)$ is the $K$-theory class of an automorphism $\tilde{\tau}(\Phi)$ of $\bigoplus_{n \in \mathbb{Z}} D_{n}$. There is an explicit formula for $\tilde{\tau}(\Phi)$ that involves the boundary map of $D_{*}, \Phi$, a chain homotopy inverse $\Phi^{\prime}$ for $\Phi$ and chain homotopies $\Phi \circ \Phi^{\prime} \rightarrow \operatorname{id}_{D_{*}}, \Phi^{\prime} \circ \Phi \rightarrow \mathrm{id}_{D_{*}}$. The ingredients for such a formula can be found for example in [2, Sec. 12.1]. A key property is that given a commutative diagram

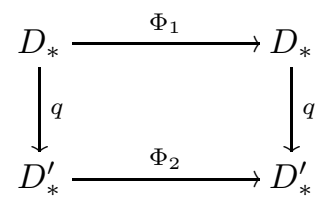

where $\Phi_{1}, \Phi_{2}$ and $q$ are chain homotopy equivalences one has $\tau\left(\Phi_{1}\right)=\tau\left(\Phi_{2}\right) \in$ $K_{1}(S)$.

Remark 4.3. It is possible to formulate Theorem 2.10 directly for self-chain homotopy equivalences of chain complexes of geometric modules of bounded dimension. Then the discussion of torsion can be avoided here. This is the point of view taken in [6. Thm. 5.3].

Step 4: $\tau(\Psi)=a$. Because $X$ is contractible, the augmentation map $C_{*} \rightarrow \mathbb{Z}$ induces a homotopy equivalence

$$
q: D_{*}=\mathbb{Z}[G] \otimes_{\mathbb{Z}} C_{*} \otimes_{\mathbb{Z}} R^{n} \rightarrow \mathbb{Z}[G] \otimes_{\mathbb{Z}} \mathbb{Z} \otimes_{\mathbb{Z}} R^{n}=\mathbb{Z}[G] \otimes_{\mathbb{Z}} R^{n} .
$$

Moreover, $q \circ \Psi=\psi \circ q$. It follows that

$$
a=[\psi]=\tau(\psi)=\tau(\Psi)=[\tilde{\tau}(\Psi)]
$$


Step 5: control of $\tilde{\tau}(\Psi)$. In order to understand the support of $\tilde{\tau}(\Psi)$ we first need to understand the support of its building blocks. If $\left(\left(h^{\prime}, x^{\prime}\right),(h, x)\right) \in(G \times X)^{2}$ belongs to the support of $\partial^{D_{*}}$, then $h^{\prime}=h$ and $d\left(x^{\prime}, x\right) \leq \delta_{0}$. If $\left(\left(h^{\prime}, x^{\prime}\right),(h, x)\right)$ belongs to the support of $\Psi$ or of its homotopy inverse $\Psi^{\prime}$, then there is $g \in T$ such that $h^{\prime}=h g^{-1}$ and $d\left(x^{\prime}, g x\right) \leq \delta_{0}$. If $\left(\left(h^{\prime}, x^{\prime}\right),(h, x)\right)$ belongs to the support of the chain homotopy $\mathcal{H}$ or $\mathcal{H}^{\prime}$ then there are $g, g^{\prime} \in T$ such that $h^{\prime}=h\left(g g^{\prime}\right)^{-1}$ and $d\left(x^{\prime}, g g^{\prime} x\right) \leq \delta_{0}$. From the explicit formula for $\tilde{\tau}(\Psi)$ one can then read off that there is a number $K$, depending only on the dimension of $D_{*}$ (which is in our case bounded by $N)$, such that the support of $\tilde{\tau}(\Psi)$ satisfies the following condition: if $\left(\left(h^{\prime}, x^{\prime}\right),(h, x)\right) \in \operatorname{supp} \tilde{\tau}(\Psi)$ then there are $g_{1}, \ldots, g_{K} \in T$ such that

$$
h^{\prime}=h\left(g_{1} \ldots g_{K}\right)^{-1} \quad \text { and } \quad d\left(x^{\prime}, g_{1} \ldots g_{K} x\right) \leq K \delta_{0} .
$$

Note that we specified $K$ in this step; note also that $K$ does only depend on $N$.

Remark 4.4. The actual value of $K$ is of course not important. It is not very large; for example $K:=10 N$ works - I think.

Step 6: applying $f$. Using the map $f: X \rightarrow E$ we define the $G$-map $F: G \times X \rightarrow$ $E$ by $F(h, x):=h f(x)$. Combining the estimates from the end of step 2 and the analysis of $\operatorname{supp}(\tilde{\tau}(\Psi))$ it is not hard to see that $\tilde{\tau}(\Psi)$ is an $\varepsilon_{N}$-automorphism over $E$ (with respect to $F$ ).

This finishes the discussion of the surjectivity of $\alpha_{\mathcal{F}}: H_{1}^{G}\left(E_{\mathcal{F}} G ; \mathbf{K}_{R}\right) \rightarrow K_{1}(R[G])$ under the assumptions of Theorem $\mathrm{A}$. Surjectivity of this map under the assumptions of Theorem $\mathrm{B}$ follows from a very similar argument; mostly step 2 is slightly more complicated. For Theorem $\mathrm{C}$ the transfer can no longer be constructed using a chain complex associated to a space; instead Swan's Theorem 3.20 is used to construct a transfer. Otherwise the proof is again very similar.

$L$-theory transfer. The proof of the $L$-theory version of Theorems $\mathrm{A}$ and $\mathrm{B}$ follows the same outline. Now elements in $L$-theory are given by quadratic forms. The analog of chain homotopy self-equivalences in $L$-theory are ultra-quadratic Poincaré complexes [42. These are chain complex versions of quadratic forms. The main difference is that to construct a transfer it is no longer sufficient to have just the chain complex $C$, in addition we need a symmetric structure on this chain complex. Moreover, this symmetric structure needs to be controlled (just as the boundary map $\partial$ is controlled). While there may be no such symmetric structure on $C$, there is a symmetric structure on the product of $C$ with its dual $D:=C \otimes C^{-*}$. This symmetric structure is given (up to signs) by $\langle a \otimes \alpha, b \otimes \beta\rangle=\alpha(b) \beta(a)$ and turns out to be suitably controlled. This is the only significant change from the proof in $K$-theory to the proof in $L$-theory.

Transfer for higher $K$-theory. We end this section with a very informal discussion of one aspect of the proof of Theorem $\mathrm{A}$ for higher $K$-theory. Again, we focus on surjectivity. In this case we use Theorem 2.20 in place of Theorem 2.10 , Thus we need to produce an element in $K_{*}(\mathcal{C})$. Recall that objects of $\mathcal{C}$ are sequences $\left(M_{\nu}\right)_{\nu \in \mathbb{N}}$ of geometric $R[G]$-modules and that morphisms are sequences of $R[G]$-linear maps that become more controlled with $\nu \rightarrow \infty$. The general idea is to apply the transfer from Step 3 to each $\nu$ to produce a functor from $R[G]$-modules to $\mathcal{C}$. The problem is, however, that the construction from Step 3 is not functorial. The reason for this in turn is that the group $G$ only acts up to homotopy on the chain complex $C_{*}$. The remedy for this failure is to use the singular chain complex of $C_{*}^{\text {sing }}(X)$ in place of $C_{*}$. It is no longer finite, but it is homotopy finite, which is finite enough. For the control consideration from Step 5 it was important, that the boundary map of $C_{*}$ is $\delta_{0}$-controlled. This is no longer true for $C_{*}^{\text {sing }}(X)$. One might be tempted to use the subcomplex $C^{\operatorname{sing}, \delta_{0}}(X)$ spanned by singular simplices 
in $X$ of diameter $\leq \delta_{0}$. However, the action of $G$ on $X$ is not isometric and therefore there is no $G$-action on $C^{\operatorname{sing}, \delta_{0}}(X)$. Finally, the solution is to use $C_{*}^{\text {sing }}(X)$ together with its filtration by the subcomplexes $\left(C_{*}^{\text {sing }, \delta}(X)\right)_{\delta>0}$. Using this idea it is possible to construct a transfer functor from the category of $R[G]$-modules to a category $\widetilde{c h}_{h f d} \mathcal{C}$. The latter is a formal enlargement of the Waldhausen category $c h_{h f d} \mathcal{C}$ of homotopy finitely dominated chain complexes over the category $\mathcal{C}[12$, Appendix]. Both the higher $K$-theory of $c h_{h f d} \mathcal{C}$ and of $\widetilde{c h}_{h f d} \mathcal{C}$ coincide with the higher $K$-theory of $\mathcal{C}$. Similar constructions are used in [9, 45.

\section{Flow SPACES}

Convention 5.1. A CAT(0)-group is a group that admits a cocompact, proper and isometric action on a finite dimensional CAT(0)-space.

The goal of this section is to outline the proof of the fact [7] that CAT(0)groups satisfy the assumptions of Theorem B. Note that CAT(0)-groups are finitely presentable [16, Thm. III.Г.1.1(1), p.439].

Proposition 5.2. Let $G$ be a CAT(0)-group. Exhibit $G$ as a finitely presented group $\langle S \mid R\rangle$. Then there is $N \in \mathbb{N}$ such that for any $\varepsilon>0$ there are

(a) an $N$-transfer space $X$ equipped with a homotopy $G$-action $(\varphi, H)$,

(b) a simplicial $(G, \mathrm{VCyc})$-complex $E$ of dimension at most $N$,

(c) a map $f: X \rightarrow E$ that is $G$-equivariant up to $\varepsilon$ : for all $x \in X, s \in S \cup S^{-1}$, $r \in R$

$-d^{1}\left(f\left(\varphi_{s}(x)\right), s \cdot f(x)\right) \leq \varepsilon$,

- $\left\{H_{r}(t, x) \mid t \in[0,1]\right\}$ has diameter at most $\varepsilon$.

An $(\alpha, \varepsilon)$-version of the assumptions of Theorem B. Let $G$ be a group.

Definition 5.3. An $N$-flow space $F S$ for $G$ is a metric space with a continuous flow $\phi: F S \times \mathbb{R} \rightarrow F S$ and an isometric proper action of $G$ such that

(a) the flow is $G$-equivariant: $\phi_{t}(g x)=g \phi_{t}(x)$ for all $x \in X, t \in \mathbb{R}$ and $g \in G$;

(b) $F S \backslash\left\{x \mid \phi_{t}(x)=x\right.$ for all $\left.t \in \mathbb{R}\right\}$ is locally connected and has covering dimension at most $N$.

Notation 5.4. Let $\alpha, \varepsilon \geq 0$. For $x, y \in F S$ we write

$$
d_{F S}^{f o l}(x, y) \leq(\alpha, \varepsilon)
$$

if there is $t \in[-\alpha, \alpha]$ such that $d\left(\phi_{t}(x), y\right) \leq \varepsilon$.

Of course, $\varepsilon$ will usually be a small number while $\alpha$ will often be much larger.

Proposition 5.5. Let $G$ be a CAT(0)-group. Exhibit $G$ as a finitely presented group $\langle S \mid R\rangle$. Then there exists $N \in \mathbb{N}$ and a cocompact $N$-flow space for $G$ and $\alpha>0$ such that for all $\varepsilon>0$ there are

(a) an $N$-transfer space $X$ equipped with a homotopy $G$-action $(\varphi, H)$,

(b) a map $f: X \rightarrow F S$ that is G-equivariant up to $(\alpha, \varepsilon)$ : for all $x \in F S$, $s \in S \cup S^{-1}, r \in R, t \in[0,1]$

$$
\begin{aligned}
& -d_{F S}^{f o l}\left(f\left(\varphi_{s}(x)\right), s \cdot f(x)\right) \leq(\alpha, \varepsilon), \\
& -d_{F S}^{f o l}\left(f\left(H_{r}(t, x)\right), f(x)\right) \leq(\alpha, \varepsilon) .
\end{aligned}
$$

The proof of Proposition 5.5 will be discussed in a later subsection. The key ingredient that allows to deduce Proposition 5.2 from Proposition [5.5] are the long and thin covers for flow spaces from 8, that in turn generalize the long and thin cell structures of Farrell-Jones [23, Sec. 7]. 
Definition 5.6. Let $R>0$. A collection $\mathcal{U}$ of open subsets of $F S$ is said to be an $R$-long cover of $A \subseteq F S$ if for all $x \in A$ there is $U \in \mathcal{U}$ such that

$$
\phi_{[-R, R]}(x):=\left\{\phi_{t}(x) \mid t \in[-R, R]\right\} \subseteq U .
$$

Notation 5.7. (Periodic orbits) Let $\gamma>0$. Write $F S_{\leq \gamma}$ for the subset of $F S$ consisting of all points $x$ for which there are $0<\tau \leq \gamma$ and $g \in G$ such that $\phi_{\tau}(x)=g x$.

Theorem 5.8 (Existence of long thin covers). Let FS be a cocompact $N$-flow space for $G$. Then there is $\tilde{N}$ such that for all $R>0$ there exists $\gamma>0$ and a collection $\mathcal{U}$ of open subsets of FS such that

(a) $\operatorname{dim} \mathcal{U} \leq \tilde{N}$ : any point of $F S$ is contained in at most $\tilde{N}+1$ members of $\mathcal{U}$,

(b) $\mathcal{U}$ is an R-long cover of $F S \backslash F S_{\leq \gamma}$,

(c) $\mathcal{U}$ is $G$-invariant: for $g \in G, U \in \mathcal{U}$ we have $g(U) \in \mathcal{U}$,

(d) $\mathcal{U}$ has finite isotropy: for all $U \in \mathcal{U}$ the group $G_{U}:=\{g \in G \mid g(U)=U\}$ is finite.

Example 5.9. Let $G:=\mathbb{Z}$. Consider $F S:=\mathbb{R}$ with the usual $\mathbb{Z}$-action and the flow defined by $\phi_{t}(x):=x+t$. If $\mathcal{U}_{R}$ is an $R$-long $\mathbb{Z}$-invariant cover of $\mathbb{R}$ of finite isotropy then the dimension of $\mathcal{U}_{R}$ grows linearly with $R$.

Theorem 5.8 states that this is the only obstruction to the existence of uniformly finite dimensional arbitrary long $G$-invariant covers of $F S$ of finite isotropy.

Remark 5.10. Theorem [5.8 is more or less [8, Thm. 1.4], see also [7, Thm. 5.6]. The proof depends only on fairly elementary constructions, but is nevertheless very long. (It would be nice to simplify this proof - but I do not know where to begin.)

In these references in addition an upper bound for the order of finite subgroups of $G$ is assumed. This assumption is removed in recent (and as of yet unpublished) work of Adam Mole and Henrik Rüping.

Remark 5.11. For the flow spaces, that have been relevant for the Farrell-Jones conjecture so far, it is possible to extend the cover $\mathcal{U}$ from $F S \backslash F S_{\leq \gamma}$ to all of $F S$. The only price one has to pay for this extension is that in assertion (d) one has to allow virtually cyclic groups instead of only finite groups. Note that with this change Example 5.9 is no longer problematic; we can simply set $\mathcal{U}_{R}:=\{\mathbb{R}\}$.

It is really at this point where the family of virtually cyclic subgroups plays a special role and appears in proofs of the Farrell-Jones Conjecture.

Remark 5.12. In the case of CAT(0) groups the extension of the cover from $F S \backslash F S_{\gamma}$ to $F S$ is really the most technical part of the arguments in [7].

It would be more satisfying to have a result that provides this extension (after allowing virtually cyclic groups) for general cocompact flow spaces.

Remark 5.13. One may think of Theorem 5.8 as a (as of now quite difficult!) parametrized version of the very easy fact that $\mathbb{Z}$ has finite asymptotic dimension.

Sketch of proof for Proposition 5.2 using Proposition 5.5. The idea is easy. We produce a map $F: F S \rightarrow E$ that is suitably contracting along the flow lines of $\phi$. Then we can compose $f: X \rightarrow F S$ from Proposition 5.5 with $F$ to produce the required map $F \circ f: X \rightarrow E$.

Let $G$ be a CAT(0)-group. Let $\varepsilon>0$ be given. Let $F S$ be the cocompact $N$-flow space for $G$ from Proposition 5.5. As discussed in Remark 5.11 there is $\tilde{N}$ such that for all $R>0$ there exists a collection $\mathcal{U}$ of open subsets of $F S$ such that

(a) $\operatorname{dim} \mathcal{U} \leq \tilde{N}$,

(b) $\mathcal{U}$ is an $R$-long cover of $F S$,

(c) $\mathcal{U}$ is $G$-invariant, 
(d) $\mathcal{U}$ has virtually cyclic isotropy: for all $U \in \mathcal{U}$ the group $G_{U}:=\{g \in G \mid$ $g(U)=U\}$ is virtually cyclic.

Let now $E:=|\mathcal{U}|$ be the nerve of the cover $\mathcal{U}$. The vertex set of this simplicial complex is $\mathcal{U}$ and we have $|\mathcal{U}|=\left\{\sum_{U \in \mathcal{U}} t_{U} U \mid t_{U} \in[0,1], \sum_{U \in \mathcal{U}} t_{U}=1\right.$ and $\bigcap_{t_{U} \neq 0} U \neq$ $\emptyset\}$. Note that $|\mathcal{U}|$ is a simplicial $(G, \mathrm{VCyc})$-complex. To construct the desired map $F: F S \rightarrow E$ we first change the metric on $F S$. For (large) $\Lambda>0$ we can define a metric that blows up the metric transversal to the flow $\phi$, and corresponds to time along flow lines. More precisely,

$$
\begin{aligned}
d_{\Lambda}(x, y):=\inf \left\{\sum_{i=1}^{n} \alpha_{i}+\Lambda \varepsilon_{i} \mid\right. & \exists x=x_{0}, x_{1}, \ldots, x_{n} \text { such that } \\
& \left.d_{F S}^{f o l}\left(x_{i-1}, x_{i}\right) \leq\left(\alpha_{i}, \varepsilon_{i}\right) \text { for } i=1, \ldots, n\right\}
\end{aligned}
$$

For $U \in \mathcal{U}, x \in F S$ let $a_{U}(x):=d_{\Lambda}(x, F S \backslash U)$ and define $F: F S \rightarrow|\mathcal{U}|$ by

$$
F(x):=\sum_{U \in \mathcal{U}} \frac{a_{U}(x)}{\sum_{V \in \mathcal{U}} a_{V}(x)} U .
$$

As $\mathcal{U}$ is $G$-invariant, $F$ is $G$-equivariant. If $R>0$ is sufficiently large (depending only on $\varepsilon$ ), then there are $\Lambda>0$ and $\delta>0$ (depending on everything at this point) such that

$$
d_{F S}^{f o l}\left(x, x^{\prime}\right) \leq(\alpha, \delta) \Longrightarrow d^{1}\left(F(x), F\left(x^{\prime}\right)\right) \leq \varepsilon .
$$

(More details for similar calculations can be found in [9, Sec. 4.3, Prop. 5.3].) Thus we can compose with $F$ and conclude that Proposition 5.5 implies Proposition 5.2 .

The flow space for a CAT(0)-space. This subsection contains an introduction to the flow space for $\operatorname{CAT}(0)$-groups from [7]. Let $Z$ be a $\operatorname{CAT}(0)$-space.

Definition 5.14. A generalized geodesic in $Z$ is a continuous map $c: \mathbb{R} \rightarrow Z$ for which there exists an interval $\left(c_{-}, c_{+}\right)$such that $\left.c\right|_{\left(c_{-}, c_{+}\right)}$is a geodesic and $\left.c\right|_{\left(-\infty, c_{-}\right)}$ and $\left.c\right|_{\left(c_{+},+\infty\right)}$ are constant. (Here $c_{-}=-\infty$ and/or $c_{+}=+\infty$ are allowed.)

Definition 5.15. The flow space for $Z$ is the space $F S(Z)$ of all generalized geodesics $c: \mathbb{R} \rightarrow Z$. It is equipped with the metric

$$
d_{F S}\left(c, c^{\prime}\right):=\int_{\mathbb{R}} \frac{d\left(c(t), c^{\prime}(t)\right)}{2 e^{|t|}} d t
$$

and the flow

$$
\phi_{\tau}(c)(t):=c(t+\tau) .
$$

Remark 5.16. The fixed point space for the flow $F S(Z)^{\mathbb{R}}:=\left\{c \mid \phi_{t}(c)=c\right.$ for all $\left.t\right\}$ is via $c \mapsto c(0)$ canonically isometric to $Z$.

The flow space $F S(Z)$ is somewhat singular around $Z=F S(Z)^{\mathbb{R}}$. For example there are well defined maps $c \mapsto c( \pm \infty)$ from $F S(Z)$ to the bordification [16, Ch.II.8] $\bar{Z}$ of $Z$, but these maps fail to be continuous at $Z$.

Remark 5.17. The metric $d_{F S}\left(c, c^{\prime}\right)$ cares most about $d\left(c(t), c^{\prime}(t)\right)$ for $t$ close to 0 . For example if $c(0)=c^{\prime}(0)$ then $d_{F S}\left(c, c^{\prime}\right)$ is bounded by $\int_{0}^{\infty} \frac{t}{e^{t}} d t$. For this reason one can think of $c(0)$ as marking the generalized geodesic $c$. If $c(0)$ is different from both $c\left(c_{-}\right)$and $c\left(c_{+}\right)$(equivalently if $c_{-}<0<c_{+}$) then the triple $\left(c\left(c_{-}\right), c(0), c\left(c_{+}\right)\right)$uniquely determines $c$.

Remark 5.18. An isometric action of $G$ on $Z$ induces an isometric action on $F S(Z)$ via $(g \cdot c)(t):=g \cdot c(t)$. If the action of $G$ on $Z$ is in addition cocompact, proper and $Z$ has dimension at most $N$, then $F S(Z)$ is a cocompact $3 N+2$-flow space for $G$ in the sense of Definition [5.3, see [7, Sec. 2]. 
For cocompactness it is important that we allowed $c_{-}=-\infty$ and $c_{+}=+\infty$ in the definition of generalized geodesics.

Remark 5.19. For hyperbolic groups there is a similar flow space constructed by Mineyev 36. This space is an essential ingredient for the proof that hyperbolic groups satisfy the assumptions of Theorem A Mineyev's construction motivated the flow space for $\mathrm{CAT}(0)$ groups.

However, for hyperbolic groups the construction is really much more difficult. A priori, there is really no local geometry associated to a hyperbolic group, hyperbolicity is just a condition on the large scale geometry and Mineyev extracts local information from this in the construction of his flow space. In contrast, for a CAT(0)-group the corresponding CAT(0)-space provides local and global geometry right from the definition.

Sketch of proof for Proposition 5.5. Let $Z$ be a finite dimensional CAT(0)space with an isometric, cocompact, proper action of the group $G$. Let $G=\left\langle S \mid R^{\prime}\right\rangle$ be a finite presentation of $G$. Pick a base point $x_{0} \in Z$. For $R>0$ let $B_{R}\left(x_{0}\right)$ be the closed ball in $Z$ of radius $R$ around $x_{0}$. This will be our transfer space. Let $\rho_{R}: Z \rightarrow B_{R}\left(x_{0}\right)$ be the closest point projection. For $x, x^{\prime} \in Z, t \in[0,1]$ we write $t \mapsto(1-t) \cdot x+t \cdot x^{\prime}$ for the straight line from $x$ to $x^{\prime}$ parametrized by constant speed $d\left(x, x^{\prime}\right)$. For $g, h \in G, t \in[0,1], x \in B_{R}\left(x_{0}\right)$ let

$$
\begin{aligned}
\varphi_{g}^{R}(x) & :=\rho_{R}(g \cdot x), \\
H_{g, h}^{R}(t, x) & :=\rho_{R}\left((1-t) \cdot g \varphi_{h}^{R}(x)+t \cdot g h x\right) .
\end{aligned}
$$

Then $H_{g, h}^{R}$ is a homotopy $\varphi_{g}^{R} \circ \varphi_{h}^{R} \rightarrow \varphi_{g h}^{R}$. This data also specifies a homotopy action $\left(\varphi^{R}, H^{R}\right)$ on $B_{R}\left(x_{0}\right)$. We will use the map $\iota_{R}: B_{R}\left(x_{0}\right) \rightarrow F S(Z)$ where $\iota_{R}(x)$ is the unique generalized geodesic $c$ in $Z$ with $c_{-}=0, c_{+}=d\left(x, x_{0}\right), c\left(c_{-}\right)=c(0)=x_{0}$ and $c\left(c_{+}\right)=x$, i.e., the generalized geodesic from $x_{0}$ to $x$ that starts at time 0 at $x_{0}$. For $T \geq 0$ let $f^{T, R}:=\phi_{T} \circ \iota_{R}: B_{R}\left(x_{0}\right) \rightarrow F S\left(x_{0}\right)$. Proposition 5.5 follows from the next Lemma; this will conclude the sketch of proof for Proposition 5.5.

Lemma 5.20. Let $\alpha:=\max _{s \in S} d\left(x_{0}, s x_{0}\right)$. For any $\varepsilon>0$ there are $T, R>0$ such that for all $x \in F S, s \in S \cup S^{-1}, r \in R^{\prime}, t \in[0,1]$ we have

$$
\begin{aligned}
& -d_{F S}^{f o l}\left(f^{T, R}\left(\varphi_{s}^{R}(x)\right), s \cdot f^{T, R}(x)\right) \leq(\alpha, \varepsilon), \\
& -d_{F S}^{f o l}\left(f^{T, R}\left(H_{r}^{R}(t, x)\right), f^{T, R}(x)\right) \leq(\alpha, \varepsilon) .
\end{aligned}
$$

Sketch of proof. We will only discuss the first inequality; the second inequality involves essentially no additional difficulties.

Let us first visualize the generalized geodesics $c:=f^{T, R}\left(\varphi_{s}^{R}(x)\right)$ and $c^{\prime}:=s$. $f^{T, R}(x)$. The generalized geodesic $c$ starts at $c\left(c_{-}\right)=x_{0}$ and ends at $c\left(c_{+}\right)=\varphi_{s}^{R}(x)$. If $d\left(x_{0}, s x\right) \leq R$, then the endpoint $\varphi_{s}^{R}(x)$ coincides with $s x$; otherwise we can prolong $c$ (as a geodesic) until it hits $s x$. If $T \leq d\left(x_{0}, \varphi_{s}^{R}(x)\right.$ then $c(0)$ is the unique point on the image of $c$ of distance $T$ from $x_{0}$, otherwise $c(0)=c\left(c_{+}\right)=\varphi_{s}^{R}(x)=$ $\rho_{R}(s x)$. The generalized geodesic $c^{\prime}$ starts at $c^{\prime}\left(c_{-}^{\prime}\right)=s x_{0}$ and ends at $c^{\prime}\left(c_{-}^{\prime}\right)=s x$. If $T \leq d\left(s x_{0}, s x\right)$, then $c^{\prime}(0)$ is the unique point on the image of $c^{\prime}$ of distance $T$ 
from $s x_{0}$, otherwise $c^{\prime}(0)=c^{\prime}\left(c_{+}^{\prime}\right)=s x$. We draw this as

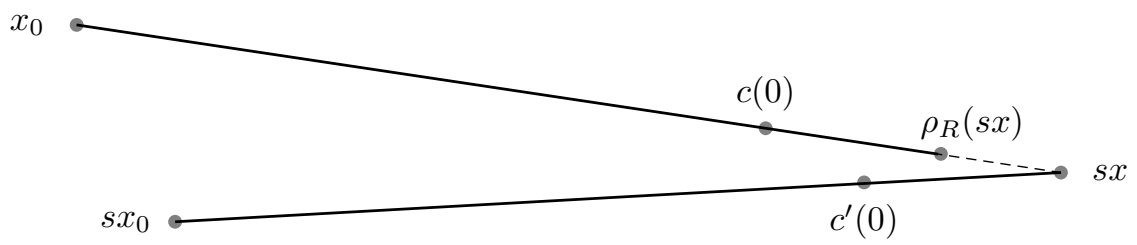

There are two basic cases to consider.

Case $I: d\left(s x, x_{0}\right)$ is small.

Then $\rho_{R}(s x)=s x$, and both $c$ and $c^{\prime}$ converge to the constant geodesic at $s x$ with $T \rightarrow \infty$. Consequently $d_{F S}\left(c, c^{\prime}\right)$ is small for large $T$.

Case II: $d\left(s x, x_{0}\right)$ is large.

Then we may have $\rho_{R}(s x) \neq s x$. Note that $d\left(\rho_{R}(s x), s x\right) \leq d\left(x_{0}, s x_{0}\right) \leq \alpha$. Let $t:=d(c(0), s x)-d\left(c^{\prime}(0), s x\right) \in[-\alpha, \alpha]$. Using the CAT $(0)$-condition one can then check that $d_{F S}\left(\phi_{t}(c), c^{\prime}\right)$ will be small provided that $T, R-T, \frac{R}{R-T}$ are large.

A more careful analysis of the two cases shows that it is possible to pick $R$ and $T$ (depending only on $\varepsilon$ ) such that for any $x$ one of the two cases applies and therefore $d_{F S}^{f o l}\left(c, c^{\prime}\right) \leq(\alpha, \varepsilon)$.

Remark 5.21. The assumption that the action of $G$ on the $\operatorname{CAT}(0)$-space $Z$ is cocompact is important for the proof of Proposition 5.2 because it implies that the action of $G$ on the flow space $F S(Z)$ is also cocompact. This in turn is important for the construction of $R$-long covers: Theorem 5.8 otherwise only allows the construction of $R$-long covers for a cocompact subspace of the flow space.

Nevertheless, there are situations where it is possible to construct $R$-long covers for flow spaces that are not cocompact. For example $\mathrm{GL}_{n}(\mathbb{Z})$ acts properly and isometrically but not cocompactly on a $\mathrm{CAT}(0)$ space. But it is possible to construct $R$-long covers for the corresponding flow space 11 . This uses as an additional input a construction of Grayson 29] and enforces a larger family of isotropy groups for the cover. This is the family $\mathcal{F}_{n-1}$ mentioned in Remark 1.9.

There are very general results of Farrell-Jones [26] without a cocompactness assumption, but I have no good understanding of these methods.

\section{6. $\mathbb{Z}^{n} \rtimes \mathbb{Z}$ AS A FARRELL-HSIANG GROUP}

For $A \in \mathrm{GL}_{n}(\mathbb{Z})$ let $\mathbb{Z}^{n} \rtimes_{A} \mathbb{Z}$ be the corresponding semi-direct product. We fix a generator $t \in \mathbb{Z}$. Then for $v \in \mathbb{Z}^{n}$ we have $t \cdot v t^{-1}=A v$ in $\mathbb{Z}^{n} \rtimes_{A} \mathbb{Z}$. The goal of this section is to outline a proof of the following fact from 3 . Recall that $\mathrm{Ab}$ denotes the family of abelian subgroups. In the case of $\mathbb{Z}^{n} \rtimes_{A} \mathbb{Z}$ these are all finitely generated free abelian.

Proposition 6.1. Suppose that no eigenvalue of $A$ over $\mathbb{C}$ is a root of unity. Then the group $\mathbb{Z}^{n} \rtimes_{A} \mathbb{Z}$ is a Farrell-Hsiang group with respect to the family $\mathrm{Ab}$ of abelian groups, i.e., there is $N$ such that for any $\varepsilon>0$ there are

(a) a group homomorphism $\pi: \mathbb{Z}^{n} \rtimes_{A} \mathbb{Z} \rightarrow F$ where $F$ is finite,

(b) a simplicial $\left(\mathbb{Z}^{n} \rtimes_{A} \mathbb{Z}, \mathrm{Ab}\right)$-complex $E$ of dimension at most $N$

(c) a map $f: \coprod_{H \in \mathcal{H}(F)} \mathbb{Z}^{n} \rtimes_{A} \mathbb{Z} / \pi^{-1}(H) \rightarrow E$ that is $\mathbb{Z}^{n} \rtimes_{A} \mathbb{Z}$-equivariant up to $\varepsilon: d^{1}(f(s x), s \cdot f(x)) \leq \varepsilon$ for all $s \in S, x \in \coprod_{H \in \mathcal{H}(F)} G / \pi^{-1}(H)$.

Here $S$ is any fixed generating set for $G$. 
Remark 6.2. The Farrell-Jones Conjecture holds for abelian groups. Thus using Theorem $\mathrm{C}$ and the transitivity principle 1.7 we deduce from Proposition 6.1 that the Farrell-Jones Conjecture holds for the group $\mathbb{Z}^{n} \rtimes_{A} \mathbb{Z}$ from Proposition 6.1.

Finite quotients of $\mathbb{Z}^{n} \rtimes_{A} \mathbb{Z}$. We write $\mathbb{Z} / s$ for the quotient ring (and underlying cyclic group) $\mathbb{Z} / s \mathbb{Z}$. Let $A_{s}$ denote the image of $A$ in $\mathrm{GL}_{n}(\mathbb{Z} / s)$. Choose $r, s \in \mathbb{N}$ such that the order $\left|A_{s}\right|$ of $A_{s}$ in $\mathrm{GL}_{n}(\mathbb{Z} / s)$ divides $r$. Then we can form $(\mathbb{Z} / s)^{n} \rtimes_{A_{s}}$ $\mathbb{Z} / r$ and there is canonical surjective group homomorphism

$$
\pi: \mathbb{Z}^{n} \rtimes_{A} \mathbb{Z} \rightarrow(\mathbb{Z} / s)^{n} \rtimes_{A_{s}} \mathbb{Z} / r .
$$

Hyper-elementary subgroups of $(\mathbb{Z} / s)^{n} \rtimes_{A_{s}} \mathbb{Z} / r$.

Lemma 6.3. Let $s=p_{1} \cdot p_{2}$ be the product of two primes. Let $r:=s \cdot\left|A_{s}\right|$. If $H$ is a hyper-elementary subgroup of $(\mathbb{Z} / s)^{n} \rtimes_{A_{s}} \mathbb{Z} / r$ then there is $q \in\left\{p_{1}, p_{2}\right\}$ such that

(a) $\pi^{-1}(H) \cap \mathbb{Z}^{n} \subseteq(q \mathbb{Z})^{n}$ or

(b) the image of $\pi^{-1}(H)$ under $\mathbb{Z}^{n} \rtimes_{A_{s}} \mathbb{Z} \rightarrow \mathbb{Z}$ is contained in $q \mathbb{Z}$.

To prove Lemma 6.3 we recall [3, Lem. 3.20].

Lemma 6.4. Let $s$ be any natural number. Let $r:=s \cdot\left|A_{s}\right|$. Let $C$ be a cyclic subgroup of $\mathbb{Z} / s^{n} \rtimes_{A_{s}} \mathbb{Z} / r$ that has nontrivial intersection with $(\mathbb{Z} / s)^{n}$.

Then there is a prime power $q^{N}(N \geq 1)$ such that

$-q^{N}$ divides $r=r^{\prime} s$,

- $q^{N}$ does not divide the order of the image of $C$ in $\mathbb{Z} / r$,

- $q$ divides the order of $C \cap(\mathbb{Z} / s)^{n}$.

Proof of Lemma 6.3. Let $H \subseteq(\mathbb{Z} / s)^{n} \rtimes_{A_{s}} \mathbb{Z} / r$ be hyper-elementary. There is a short exact sequence $C \longmapsto H \rightarrow P$ with $P$ a $p$-group and $C$ a cyclic group. The cyclic group $C$ can always be arranged to be of order prime to $p$.

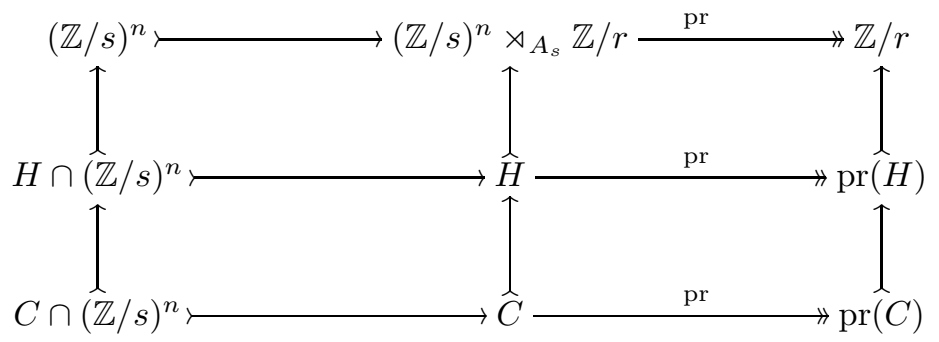

There are two cases.

Case $I: C \cap(\mathbb{Z} / s)^{n}$ is trivial.

Then $H \cap(\mathbb{Z} / s)^{n}$ is a $p$-group. Let $q$ be the prime from $\left\{p_{1}, p_{2}\right\}$ that is different from $p$. Then (a) will hold.

Case II: $C \cap(\mathbb{Z} / s)^{n}$ is nontrivial.

Then there is a prime $q$ as in Lemma 6.4. As $q$ divides $\left|C \cap(\mathbb{Z} / s)^{n}\right|$ we have $q \in\left\{p_{1}, p_{2}\right\}$ and $q \neq p$. It follows that $q$ divides $[\mathbb{Z} / r: \operatorname{pr}(H)]$. This implies (b).

\section{Contracting maps.}

Example 6.5. Consider the standard action of $\mathbb{Z}^{n}$ on $\mathbb{R}^{n}$. Let $\bar{H}:=(l \mathbb{Z})^{n} \subseteq \mathbb{Z}^{n}$ and $\varphi: \bar{H} \rightarrow \mathbb{Z}^{n}$ be the isomorphism $v \mapsto \frac{v}{l}$. Let $\operatorname{res}_{\varphi} \mathbb{R}^{n}$ be the $\bar{H}$-space obtained by restricting the action of $\mathbb{Z}^{n}$ on $\mathbb{R}^{n}$ with $\varphi_{l}$. Then $x \mapsto \frac{x}{l}$ defines an $\bar{H}$-map $F: \mathbb{Z}^{n} \rightarrow \operatorname{res}_{\varphi} \mathbb{R}^{n}$. This map is contracting. In fact by increasing $l$ we can make $F$ as contracting as we like, while we can keep the metric on $\mathbb{R}^{n}$ fixed.

A variant of this simple construction will be used to produce maps as in (c) of Proposition 6.1. This will finish the discussion of the proof of Proposition 6.1. 
Proposition 6.6. Let $S \subseteq \mathbb{Z}^{n} \rtimes_{A} \mathbb{Z}$ be finite. For any $\varepsilon>0$ there is $l_{0}$ such that for all $l \geq l_{0}$ the following holds.

Let $\bar{H}:=\mathbb{Z}^{n} \rtimes_{A}(l \mathbb{Z}) \subseteq \mathbb{Z}^{n} \rtimes_{A} \mathbb{Z}$. Then there is a simplicial $\left(\mathbb{Z}^{n} \rtimes_{A} \mathbb{Z}, \mathrm{Ab}\right)$-complex $E$ of dimension 1 and an $\bar{H}$-equivariant map

$$
F: \mathbb{Z}^{n} \rtimes_{A} \mathbb{Z} \rightarrow E
$$

such that $d^{1}(F(g), F(h)) \leq \varepsilon$ whenever $g^{-1} h \in S$.

Proof. We apply the construction of Example 6.5 to the quotient $\mathbb{Z}$ of $\mathbb{Z}^{n} \rtimes_{A} \mathbb{Z}$.

Let $E:=\mathbb{R}$. We use the standard way of making $E=\mathbb{R}$ a simplicial complex in which $\mathbb{Z} \subseteq \mathbb{R}$ is the set of vertices. Let $\bar{H}$ act on $E$ via $\left(v t^{k}\right) \cdot \xi:=\frac{k}{l} \xi$; this is a simplicial action. Finally define $F: \mathbb{Z}^{n} \rtimes_{A} \mathbb{Z} \rightarrow E$ by $F\left(v t^{k}\right):=\frac{k}{l}$. It is very easy to check that $F$ has the required properties for sufficiently large $l$.

Proposition 6.7. Let $S \subseteq \mathbb{Z}^{n} \rtimes_{A} \mathbb{Z}$ be finite. There is $N \in \mathbb{N}$ depending only on $n$ such that for any $\varepsilon>0$ there is $l_{0}$ such that for all $l \geq l_{0}$ the following holds.

Let $\bar{H}:=(l \mathbb{Z})^{n} \rtimes_{A} \mathbb{Z} \subseteq \mathbb{Z}^{n} \rtimes_{A} \mathbb{Z}$. Then there is a simplicial $\left(\mathbb{Z}^{n} \rtimes_{A} \mathbb{Z}, \mathrm{Cyc}\right)$ complex $E$ of dimension at most $N$ and an $\bar{H}$-equivariant map

$$
F: \mathbb{Z}^{n} \rtimes_{A} \mathbb{Z} \rightarrow E
$$

such that $d^{1}(F(g), F(h)) \leq \varepsilon$ whenever $g^{-1} h \in S$.

Sketch of proof. As in the proof of Proposition 6.6 we start with the construction from Example 6.5. now applied to the subgroup $\mathbb{Z}^{n} \subseteq \mathbb{Z}^{n} \rtimes_{A} \mathbb{Z}$. However, unlike the quotient $\mathbb{Z}$, there is no homomorphism from $\mathbb{Z}^{n} \rtimes_{A} \mathbb{Z}$ to the subgroup and it will be more difficult to finish the proof.

Let $\mathbb{Z}^{n} \rtimes_{A} \mathbb{Z}$ act on $\mathbb{R}^{n} \times \mathbb{R}$ via $v t^{k} \cdot(x, \xi):=\left(v+A^{k}(x), k+\xi\right)$. Let $\varphi: \bar{H} \rightarrow$ $\mathbb{Z}^{n} \rtimes_{A} \mathbb{Z}$ be the isomorphism $v t^{k} \mapsto \frac{v}{l} t^{k}$. The map $F_{0}: \mathbb{Z}^{n} \rtimes_{A} \mathbb{Z} \rightarrow \operatorname{res}_{\varphi} \mathbb{R}^{n} \times \mathbb{R}$, $\left(v t^{k}\right) \mapsto(v / l, k)$ is $\bar{H}$-equivariant and contracting in the $\mathbb{Z}^{n}$-direction, but not in the $\mathbb{Z}$-direction. In order to produce a map that is also contracting in the $\mathbb{Z}$-direction we use the flow methods from Section 5 .

There is $\mathbb{Z}^{n} \rtimes_{A} \mathbb{Z}$-equivariant flow on $\mathbb{R}^{n} \times \mathbb{R}$ defined by $\phi_{\tau}(x, \xi)=(x, \tau+\xi)$. It is possible to produce a simplicial $\left(\mathbb{Z}^{n} \rtimes_{A} \mathbb{Z}\right.$, Cyc)-complex $E$ of uniformly bounded dimension (depending only on $n$ ) and $\mathbb{Z}^{n} \rtimes_{A} \mathbb{Z}$-equivariant map $F_{1}: \mathbb{R}^{n} \times \mathbb{R} \rightarrow E$ that is contracting in the flow direction (but expanding in the transversal $\mathbb{R}^{n}$ direction). To do so one uses Theorem 5.8 $E$ will be the nerve of a suitable long cover of $\mathbb{R}^{n} \times \mathbb{R}$.

The fact that $F_{1}$ is expanding in the $\mathbb{R}^{n}$-direction can be countered by the contracting property of $F_{0}$. All together, the composition $F_{1} \circ F_{0}: \mathbb{Z}^{n} \rtimes_{A} \mathbb{Z} \rightarrow \operatorname{res}_{\varphi} E$ has the desired properties.

Remark 6.8. As many other things, the idea of using a flow space in the proof of Proposition 6.7 originated in the work of Farrell and Jones 24]. I found this trick very surprising when I first learned about it.

Lemma 6.9. Let $\bar{H}$ be a subgroup of $\mathbb{Z}^{n} \rtimes_{A} \mathbb{Z}$ and $l, k \in \mathbb{N}$ such that

(a) $\bar{H} \cap \mathbb{Z}^{n} \subseteq l \mathbb{Z}$,

(b) $\bar{H}$ maps to $k \mathbb{Z}$ under the projection $\mathbb{Z}^{n} \rtimes_{A} \mathbb{Z} \rightarrow \mathbb{Z}$,

(c) the index $\left[\mathbb{Z}^{n}:\left(\mathrm{id}-A^{k}\right) \mathbb{Z}^{n}\right]$ is finite and $l \equiv 1 \operatorname{modulo}\left[\mathbb{Z}^{n}:\left(\mathrm{id}-A^{k}\right) \mathbb{Z}^{n}\right]$.

Then $\bar{H}$ is subconjugated to $(l \mathbb{Z})^{n} \rtimes_{A} \mathbb{Z}$.

Proof. Consider the image $\bar{H}_{l}$ of $\bar{H}$ under $\mathbb{Z}^{n} \rtimes_{A} \mathbb{Z} \rightarrow(\mathbb{Z} / l)^{n} \rtimes_{A} \mathbb{Z}$. Then (a) implies that the restriction of the projection $(\mathbb{Z} / l)^{n} \rtimes_{A} \mathbb{Z} \rightarrow \mathbb{Z}$ to $\bar{H}_{l}$ is injective. In particular $\bar{H}_{l}$ is cyclic. By (b) there is $v \in \mathbb{Z}^{n}$ such that $v t^{k} \in \mathbb{Z}^{n} \rtimes_{A} \mathbb{Z}$ maps to a generator of $\bar{H}_{l}$. Assumption (c) implies that there is $w \in \mathbb{Z}^{n}$ such that 
$v \equiv\left(\mathrm{id}-A^{k}\right) w$ modulo $(l \mathbb{Z})^{n}$. A calculation shows that $w$ conjugates $\bar{H}$ to a subgroup of $(l \mathbb{Z})^{n} \rtimes_{A} \mathbb{Z}$.

Proof of Proposition 6.1. Let $L$ be a large number. Since $A$ has no roots of unity as eigenvalues, the index $i_{k}:=\left[\mathbb{Z}^{n}:\left(\mathrm{id}-A^{k}\right) \mathbb{Z}^{n}\right]$ is finite for all $k$. Let $K:=i_{1} \cdot i_{2} \cdots i_{L}$. By a theorem of Dirichlet there are infinitely many primes congruent to 1 modulo $K$. Let $s=p_{1} \cdot p_{2}$ be the product of two such primes, both $\geq L$, and set $r:=s \cdot\left|A_{s}\right|$.

We use the group homomorphism $\pi: \mathbb{Z}^{n} \rtimes_{A} \mathbb{Z} \rightarrow(\mathbb{Z} / s)^{n} \rtimes_{A_{s}} \mathbb{Z} / r$. Because of Lemma 6.3 we find for any hyper-elementary subgroup $H$ of $(\mathbb{Z} / s)^{n} \rtimes_{A_{s}} \mathbb{Z} / r$ an $q \in\left\{p_{1}, p_{2}\right\}$ such that $\pi^{-1}(H) \subseteq \mathbb{Z}^{n} \rtimes_{A}(q \mathbb{Z})$ or $\pi^{-1}(H) \cap \mathbb{Z}^{n} \subseteq(q \mathbb{Z})^{n}$. In the first case we set $l:=q$. In the second case we have either $\pi^{-1}(\bar{H}) \subseteq \mathbb{Z}^{n} \rtimes_{A}(l \mathbb{Z})$ for some $l>L$ or we can apply Lemma 6.9 to deduce that (up to conjugation) $\pi^{-1}(H) \subseteq(q \mathbb{Z})^{n} \rtimes_{A} \mathbb{Z}$ and we again set $l:=q$.

Therefore it suffices to find simplicial $\left(\mathbb{Z}^{n} \rtimes_{A} \mathbb{Z}, \mathrm{Ab}\right)$-complexes $E_{1}, E_{2}$ whose dimension is bounded by a number depending only on $n$ (and not on $l$ ) and maps

$$
\begin{array}{lll}
f_{1}: & \mathbb{Z}^{n} \rtimes_{A} \mathbb{Z} /(l \mathbb{Z})^{n} \rtimes_{A} \mathbb{Z} & \rightarrow E_{1} \\
f_{2}: & \mathbb{Z}^{n} \rtimes_{A} \mathbb{Z} / \mathbb{Z}^{n} \rtimes_{A}(l \mathbb{Z}) \rightarrow E_{2}
\end{array}
$$

that are $G$-equivariant up to $\varepsilon$. If $f: \mathbb{Z}^{n} \rtimes_{A} \mathbb{Z} \rightarrow E$ is the map from Proposi-

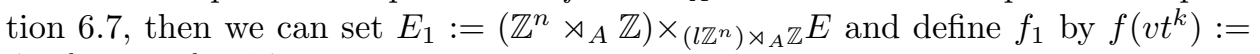
$\left(\left(v t^{k}\right), f\left(\left(v t^{k}\right)^{-1}\right)\right)$. Similarly, we can produce $f_{2}$ using Proposition 6.6.

\section{REFERENCES}

[1] A. Bartels, S. Echterhoff, and W. Lück. Inheritance of isomorphism conjectures under colimits. In Cortinaz, Cuntz, Karoubi, Nest, and Weibel, editors, K-Theory and noncommutative geometry, EMS-Series of Congress Reports, pages 41-70. European Mathematical Society, 2008.

[2] A. Bartels, T. Farrell, L. Jones, and H. Reich. On the isomorphism conjecture in algebraic K-theory. Topology, 43(1):157-213, 2004.

[3] A. Bartels, T. Farrell, and W. Lück. The Farrell-Jones Conjecture for cocompact lattices in virtually connected Lie groups. arXiv:1101.0469v1 [math.GT], 2011.

[4] A. Bartels and W. Lück. On twisted group rings with twisted involutions, their module categories and L-theory. In Cohomology of groups and algebraic K-theory, volume 12 of Advanced Lectures in Mathematics, pages 1-55, Somerville, U.S.A., 2009. International Press.

[5] A. Bartels and W. Lück. The Farrell-Hsiang method revisited. preprint, arXiv:1101.0466v1 [math.GT], to appear in Math. Annalen, 2011.

[6] A. Bartels and W. Lück. The Borel conjecture for hyperbolic and CAT(0)-groups. Ann. of Math. (2), 175:631-689, 2012.

[7] A. Bartels and W. Lück. Geodesic flow for CAT(0)-groups. Geom. Topol., 16:1345-1391, 2012.

[8] A. Bartels, W. Lück, and H. Reich. Equivariant covers for hyperbolic groups. Geom. Topol., 12(3):1799-1882, 2008.

[9] A. Bartels, W. Lück, and H. Reich. The $K$-theoretic Farrell-Jones conjecture for hyperbolic groups. Invent. Math., 172(1):29-70, 2008.

[10] A. Bartels, W. Lück, and H. Reich. On the Farrell-Jones Conjecture and its applications. Journal of Topology, 1:57-86, 2008.

[11] A. Bartels, W. Lück, H. Reich, and H. Rüping. $K$ - and $L$-theory of group rings over $G L_{n}(\mathbb{Z})$. Preprint, arXiv:1204.2418v1 [math.KT], 2012.

[12] A. Bartels and H. Reich. On the Farrell-Jones conjecture for higher algebraic $K$-theory. $J$. Amer. Math. Soc., 18(3):501-545, 2005.

[13] A. Bartels and H. Reich. Coefficients for the Farrell-Jones Conjecture. Adv. Math., 209(1):337-362, 2007.

[14] A. C. Bartels. Squeezing and higher algebraic $K$-theory. K-Theory, 28(1):19-37, 2003.

[15] M. Bökstedt, W. C. Hsiang, and I. Madsen. The cyclotomic trace and algebraic $K$-theory of spaces. Invent. Math., 111(3):465-539, 1993.

[16] M. R. Bridson and A. Haefliger. Metric spaces of non-positive curvature. Springer-Verlag, Berlin, 1999. Die Grundlehren der mathematischen Wissenschaften, Band 319.

[17] G. Carlsson and E. K. Pedersen. Controlled algebra and the Novikov conjectures for $K$ - and L-theory. Topology, 34(3):731-758, 1995. 
[18] T. A. Chapman. Homotopy conditions which detect simple homotopy equivalences. Pacific J. Math., 80(1):13-46, 1979.

[19] T. A. Chapman and S. C. Ferry. Approximating homotopy equivalences by homeomorphisms. Amer. J. Math., 101(3):583-607, 1979.

[20] E. H. Connell and J. Hollingsworth. Geometric groups and Whitehead torsion. Trans. Amer. Math. Soc., 140:161-181, 1969.

[21] J. F. Davis and W. Lück. Spaces over a category and assembly maps in isomorphism conjectures in $K$ - and $L$-theory. $K$-Theory, 15(3):201-252, 1998.

[22] F. T. Farrell and W. C. Hsiang. The topological-Euclidean space form problem. Invent. Math., 45(2):181-192, 1978.

[23] F. T. Farrell and L. E. Jones. K-theory and dynamics. I. Ann. of Math. (2), 124(3):531-569, 1986.

[24] F. T. Farrell and L. E. Jones. The surgery L-groups of poly-(finite or cyclic) groups. Invent. Math., 91(3):559-586, 1988.

[25] F. T. Farrell and L. E. Jones. Isomorphism conjectures in algebraic K-theory. J. Amer. Math. Soc., 6(2):249-297, 1993.

[26] F. T. Farrell and L. E. Jones. Rigidity for aspherical manifolds with $\pi_{1} \subset G L_{m}(\mathbb{R})$. Asian J. Math., 2(2):215-262, 1998.

[27] F. T. Farrell and P. A. Linnell. K-theory of solvable groups. Proc. London Math. Soc. (3), 87(2):309-336, 2003.

[28] S. C. Ferry. The homeomorphism group of a compact Hilbert cube manifold is an anr. Ann. Math. (2), 106(1):101-119, 1977.

[29] D. R. Grayson. Reduction theory using semistability. Comment. Math. Helv., 59(4):600-634, 1984.

[30] E. Guentner, R. Tessera, and G. Yu. A notion of geometric complexity and its application to topological rigidity. Invent. Math., 189(2):315-357, 2012.

[31] W. Lück. Survey on classifying spaces for families of subgroups. In Infinite groups: geometric, combinatorial and dynamical aspects, volume 248 of Progr. Math., pages 269-322. Birkhäuser, Basel, 2005.

[32] W. Lück. On the Farrell-Jones Conjecture and related conjectures. In Cohomology of groups and algebraic K-theory, volume 12 of Advanced Lectures in Mathematics, pages 269-341, Somervile, U.S.A., 2009. International Press.

[33] W. Lück. Survey on aspherical manifolds. In A. Ran, H. te Riele, and J. Wiegerinck, editors, Proceedings of the 5-th European Congress of Mathematics Amsterdam 14 -18 July 2008, pages 53-82. EMS, 2010.

[34] W. Lück. $K$ - and $L$-theory of group rings. In R. Bhatia, editor, Proceedings of the 26-th ICM in Hyderabad, August 19-27, 2010, volume II, pages 1071-1098. World Scientific, 2011.

[35] W. Lück and H. Reich. The Baum-Connes and the Farrell-Jones conjectures in $K$ - and $L$ theory. In Handbook of K-theory. Vol. 1, 2, pages 703-842. Springer, Berlin, 2005.

[36] I. Mineyev. Flows and joins of metric spaces. Geom. Topol., 9:403-482 (electronic), 2005.

[37] E. K. Pedersen. Controlled algebraic K-theory, a survey. In Geometry and topology: Aarhus (1998), volume 258 of Contemp. Math., pages 351-368. Amer. Math. Soc., Providence, RI, 2000.

[38] E. K. Pedersen and C. A. Weibel. K-theory homology of spaces. In Algebraic topology (Arcata, $C A, 1986)$, pages 346-361. Springer-Verlag, Berlin, 1989.

[39] F. Quinn. Ends of maps. I. Ann. of Math. (2), 110(2):275-331, 1979.

[40] F. Quinn. Algebraic K-theory over virtually abelian groups. J. Pure Appl. Algebra, 216(1):170-183, 2012.

[41] D. Ramras, R. Tessera, and G. Yu. Finite decomposition complexity and the integral Novikov conjecture for higher algebraic K-theory. Preprint, arXiv:1111.7022 v2 [math.KT], 2011.

[42] A. A. Ranicki. Algebraic L-theory and topological manifolds. Cambridge University Press, Cambridge, 1992.

[43] R. G. Swan. Induced representations and projective modules. Ann. of Math. (2), 71:552-578, 1960.

[44] J. B. Wagoner. Delooping classifying spaces in algebraic K-theory. Topology, 11:349-370, 1972.

[45] C. Wegner. The $K$-theoretic Farrell-Jones conjecture for CAT(0)-groups. Proc. Amer. Math. Soc., 140(3):779-793, 2012.

[46] G. Yu. The coarse Baum-Connes conjecture for spaces which admit a uniform embedding into Hilbert space. Invent. Math., 139(1):201-240, 2000. 
Westfälische Wilhelms-Universität MÜnster, Mathematisches Institut, Einsteinstr. 62, D-48149 MÜnster, Germany

E-mail address: a.bartels@uni-muenster.de

$U R L:$ http://www.math.uni-muenster.de/u/bartelsa 